\title{
Dynamical $x$-ray diffraction of multilayers and superlattices: Recursion matrix extension to grazing angles
}

\author{
S. A. Stepanov, ${ }^{*}$ E. A. Kondrashkina, ${ }^{*}$ and R. Köhler \\ $A G$ “Röntgenbeugung," Humboldt University of Berlin, Hausvogteiplatz 5-7, Berlin 10117, Germany \\ D. V. Novikov and G. Materlik \\ HASYLAB at DESY, Notkestrasse 85, Hamburg 22603, Germany
}

S. M. Durbin

Department of Physics, Purdue University, West Lafayette, Indiana 47907

(Received 7 February 1997)

\begin{abstract}
A generalized dynamical theory has been developed that extends previous models of x-ray diffraction from crystals and multilayers with vertical strains to the cases of grazing incidence and/or exit below the critical angle for total specular reflection. This provides a common description for extremely asymmetric diffraction, surface ("grazing-incidence"), and grazing Bragg-Laue diffraction, thus providing opportunities for the applications of grazing geometries to the studies of thin multilayers. The solution, obtained in the form of recursion formulas for $(2 \times 2)$ scattering matrices for each individual layer, eliminates possible divergences of the $(4 \times 4)$ transfer-matrix algorithm developed previously. For nongrazing $x$-ray diffraction in the Bragg geometry and for grazing-incidence $\mathrm{x}$-ray specular reflection out of the Bragg diffraction conditions, the matrices are reduced to scalars and the recursion formulas become equivalent to the earlier recursion formulas by Bartels et al. [Acta Cryst. A 42, 539 (1986)] and Parratt [Phys. Rev. 95, 359 (1954)], respectively. The theory has been confirmed by an extremely asymmetric x-ray-diffraction experiment with a strained AlAs/GaAs superlattice carried out at HASYLAB. A solution to the difficulties due to dispersion encountered in extremely asymmetric diffraction measurements has been demonstrated. Finally, the validity of Ewald's expansion for thin layers and the relation of the matrix method to the Darwin theory, as well as the structure of x-ray standing waves in multilayers are discussed. [S0163-1829(98)05408-3]
\end{abstract}

\section{INTRODUCTION}

In recent years $\mathrm{x}$-ray diffraction schemes with grazing incidence and/or exit angles have attracted particular interest because of their advantages in the studies of very thin surface layers of crystals. ${ }^{1-4}$ Due to the total external reflection effect for grazing $\mathrm{x}$ rays, the $\mathrm{x}$-ray penetration inside crystals is reduced from the micrometer range down to a few nanometers, thus providing the possibility of studying surface structures with atomic depth resolution.

Grazing geometries can be classified into three major types (Fig. 1): (i) The coplanar extremely asymmetric diffraction $(E A D)$ is realized when the diffraction planes make the Bragg angle with the crystal surface and either the incident or exit $\mathrm{x}$-ray wave is grazing. ${ }^{5-12}$ (ii) Surface or "grazing-incidence", diffraction ${ }^{13}$ (GID) is the geometry where the Bragg planes are perpendicular to the surface and both the X-ray waves are grazing. ${ }^{14-23}$ (iii) Finally, grazing Bragg-Laue diffraction (GBL) is a combination of the EAD and GID. It involves the diffraction from atomic planes inclined at a small angle to the crystal surface normal, so that the reciprocal lattice vector points outside the crystal at few degrees to the surface. It is then possible to choose asymmetric diffraction with either grazing incidence or grazing exit by a small variation in the incidence angle or even to switch between these two cases within one diffraction experiment. $^{24,25}$

All three geometries have found widespread application in studies of semiconductor crystal surface structures, including diffusion-induced, ion-implanted, and epitaxial layers and multilayers, oxidized, etched, and corrugated surfaces, etc. (see Refs. 26-37, Refs. 15,38-51, and Refs. 52-54 for EAD, GID, and GBL, respectively). However, these studies also indicated the lack of a general diffraction model for the various grazing geometries.

X-ray diffraction at grazing incidence and/or exit can be treated with the help of either an extended kinematical theory (often called the "distorted wave Born approximation', ), ${ }^{16,19,41,49,55}$ or extended dynamical theory. $5,6,8,9,11,14,17,22,45,48,51,56-59$ Both approaches take into account refraction and specular reflection effects for grazing $\mathrm{x}$ rays at crystal surfaces and interfaces. As with ordinary Bragg diffraction, the kinematical theory is applicable to mosaic crystals, to the tails of the Bragg peaks, and to the diffraction from layers thinner than the x-ray extinction depth. This depth decreases to about $10 \mathrm{ML}$ under total external reflection conditions for grazing $\mathrm{x}$ rays. The application of the perturbation (kinematical) theory to the above-listed cases is possible due to a small intensity of diffracted $\mathrm{x}$ rays. Otherwise the dynamical theory must be applied, which takes into account comparable intensities of incident and diffracted waves and their multiple rescattering into each other.

Improvement in the dynamical theory is demanded by the steadily improving quality and increasing complexity of semiconductor heterostructures. However, the majority of theoretical studies have been concerned with perfect crystals 

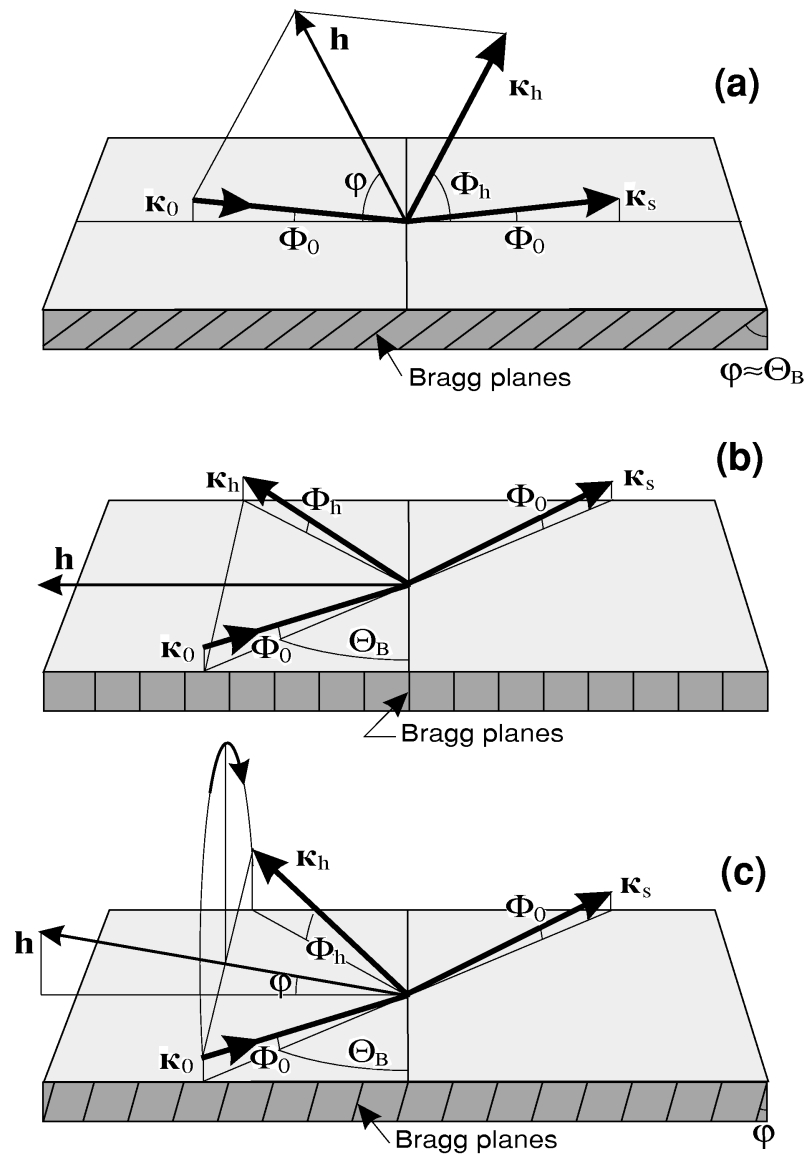

FIG. 1. Diffraction geometries with $\mathrm{x}$-ray grazing incidence and/or exit. (a) coplanar extremely asymmetric diffraction (grazingincidence case), (b) grazing-incidence diffraction, and (c) grazing Bragg-Laue diffraction. Vectors $\boldsymbol{\kappa}_{0}, \boldsymbol{\kappa}_{\mathrm{s}}$, and $\boldsymbol{\kappa}_{\mathrm{h}}$ denote incident, specularly reflected, and diffracted waves, respectively; $\boldsymbol{h}$ is the reciprocal lattice vector corresponding to the Bragg planes, $\Phi_{0}$, $\Phi_{\mathrm{h}}$, and $\varphi$ are the angles of $\boldsymbol{\kappa}_{0}, \boldsymbol{\kappa}_{\mathrm{h}}$, and $\boldsymbol{h}$, respectively, with the surface; $\theta_{\mathrm{B}}$ is the Bragg angle.

or with greatly simplified models of defect crystals. The problem is that the diffraction from strained crystals in the case of grazing incidence/exit cannot be analyzed within the standard Takagi-Taupin approach, ${ }^{60,61}$ which is based on the assumption that the $\mathrm{x}$-ray wave-field amplitudes vary slowly at interatomic distances so that their second derivatives can be neglected. A new general theory applicable to x-ray diffraction with grazing incidence and/or exit was constructed in a $(4 \times 4)$ matrix form. ${ }^{45,48,51,56-59}$ (In Refs. 56 and 57 the rank of matrices was $8 \times 8$ because $\sigma$ - and $\pi$-X-ray polarizations were treated together.) The two different formulations discussed in these papers, i.e., the differential matrix equations $s^{56,57}$ and the algebraic equations for transfer matrices, ${ }^{45,51,48,58,59}$ are essentially equivalent. The former approach is more convenient for continuous strain profiles in crystals, while the latter one is superior for multilayers. This transfer matrix $(\mathrm{TM})$ technique is similar to the $(2 \times 2)$ matrix solution for grazing incidence $\mathrm{x}$-ray specular reflection of multilayers found by Abeles. ${ }^{62}$

Unfortunately, both the differential and algebraic versions of the $(4 \times 4)$ matrix technique may suffer from serious numerical problems in their computer implementation. In addition, the TM theory makes use of Ewald's expansion for
$\mathrm{X}$-ray wave fields in an infinite crystal, whose applicability to thin layers must be established. The aim of the present paper is to reformulate the matrix dynamical theory in a recursion matrix (RM) form that overcomes the numerical problems of the TM formulation, and to provide an experimental check to the Ewald expansion for thin strained multilayers.

In Sec. II the $(4 \times 4)$ transfer matrix theory ${ }^{48,58,59}$ of $x$-ray diffraction from multilayers is outlined, and in Sec. III possible numerical problems are demonstrated and explained.

In Sec. IV the theory is reformulated in terms of recursion formulas for $(2 \times 2)$ matrices for individual layers. It is shown how this approach overcomes the numerical problems of the $(4 \times 4)$ TM algorithm. In Sec. V the reduction of the matrix recursion formulas to the scalar recursion formulas by Parratt ${ }^{63}$ and Bartels, Hornstra and Lobeek ${ }^{64}$ is demonstrated for $\mathrm{x}$-ray grazing incidence far from the Bragg diffraction condition and for Bragg diffraction with no grazing waves.

In Sec. VI the recursion matrix theory is verified with the help of the double-crystal EAD measurements taken from a strained AlAs/GaAs superlattice (SL) with known structure parameters.

In Sec. VII the results of recursion matrix calculations are compared to those given by the Darwin theory applied to heterostructure diffraction. ${ }^{65}$ In contrast to our approach, the Darwin theory does not contain Ewald's expansion and directly sums x-ray scattering of atomic planes. It is shown that in the case of ordinary Bragg diffraction the results of both theories coincide. Further comparisons require an extension of the Darwin theory to grazing angles, which is forthcoming. ${ }^{66}$ In Sec. IX the structure of x-ray standing waves in multilayers is discussed.

We conclude with some possible uses and further extensions of the recursion matrix theory.

\section{II. $(4 \times 4)$ MATRIX THEORY OF MULTILAYER DIFFRACTION}

Let us consider x-ray Bragg diffraction in one of the geometries presented in Fig. 1. The crystal is assumed to be a multilayer consisting of a stack of $N$ perfect crystalline layers with laterally matched lattice spacing. We allow each layer to possess its own lattice spacing $a_{z}^{n}$ in the direction normal to the surface: $a_{z}^{n}=a_{z}+\Delta a_{z}^{n}$, where $\left|\Delta a_{z}^{n}\right| \ll a_{z}$, and $n$ is the layer index numbered from the surface of the stack. This model corresponds to a so-called unrelaxed multilayer containing no misfit dislocations. Methods to extend this model to multilayers with misfits are briefly discussed in the Conclusions (this problem is not completely solved yet). A possibility of crystal curvature as a result of strains ${ }^{67}$ is also disregarded. That is, we assume that either the multilayer is thin enough or the substrate is thick enough to ignore curvature.

Those structures that contain additional amorphous layers are not considered here for the reason of simplicity, although they can be readily included in the model. ${ }^{48,58}$ For the same reason, we neglect possible changes in x-ray polarization, which may occur due to the refraction effects in GID and GBL. The intermixing of $\sigma$ and $\pi$ polarizations was taken into consideration, for example, in Refs. 56 and 57, but this effect is shown to be small. ${ }^{68}$ Our derivations below are car- 
ried out for $\sigma$ polarization. The equations can be extended for $\pi$ polarization by incorporating $\cos \left(2 \theta_{\mathrm{B}}\right)$ in the $\mathrm{x}$-ray susceptibilities $\chi_{\mathrm{h}}$ and $\chi_{\overline{\mathrm{h}}}$.

When Bragg diffraction from atomic planes in a ML with variable $a_{z}^{n}$ is considered, the reciprocal vector $\boldsymbol{h}_{n}$ associated with the atomic planes in the $n$th layer slightly differs from the mean vector $\boldsymbol{h}$ because of the deviation of the normal lattice spacing in the layer: $\boldsymbol{h}_{n}=\boldsymbol{h}+\Delta h_{z n} \boldsymbol{Z}$, where $\left|\Delta h_{z n}\right| \ll h$, and $\boldsymbol{Z}$ is a unit vector along the internal surface normal. We assume the local crystal dielectric susceptibility in each layer to have the same periodicity as the local atomic planes spacing in the layer:

$$
\chi^{n}(\boldsymbol{r})=\chi_{0}^{n}+\chi_{\mathrm{h}_{n}}^{n} e^{i \boldsymbol{h}_{n} \cdot\left(\boldsymbol{r}-\boldsymbol{r}_{n}\right)+i \phi_{n}}+\chi_{\mathrm{h}_{n}}^{n} e^{-i \boldsymbol{h}_{n} \cdot\left(\boldsymbol{r}-\boldsymbol{r}_{n}\right)-i \phi_{n}},
$$

where the beginning of coordinates $\boldsymbol{r}_{n}$ is taken at the upper layer interface, and the initial phase $\phi_{n}$ will be chosen later. For small strains the Fourier coefficients $\chi_{\mathrm{h}_{n}}^{n}, \chi_{\mathrm{h}_{n}}^{n}$ can be set equal to the Fourier coefficients $\chi_{\mathrm{h}}^{n}, \chi_{\mathrm{h}}^{n}$ of unstrained crystal. $^{60}$

Following the standard Ewald approach to dynamical diffraction theory, we expand the x-ray wave field in each layer over the sum of the transmitted and diffracted Bloch waves with wave vectors $\boldsymbol{k}_{0 n}$ and $\boldsymbol{k}_{\mathrm{h} n}=\boldsymbol{k}_{0 n}+\boldsymbol{h}_{n}$, and amplitudes $D_{0 n}$ and $D_{\mathrm{h} n}$, respectively: ${ }^{69-7}$

$$
D_{n}(\boldsymbol{r})=e^{i \boldsymbol{k}_{0 n} \cdot \boldsymbol{r}}\left[D_{0 n}+D_{\mathrm{h} n} e^{i \boldsymbol{h}_{n} \cdot\left(\boldsymbol{r}-\boldsymbol{r}_{n}\right)+i \phi_{n}}\right] .
$$

Under the expansions (1) and (2), the amplitudes $D_{0 n}$ and $D_{\mathrm{h} n}$ can be treated as constants satisfying the dynamical diffraction equations in each layer:

$$
\begin{aligned}
& \frac{k_{0 n}^{2}-\kappa_{0}^{2}}{k_{0 n}^{2}} D_{0 n}=\chi_{0}^{n} D_{0 n}+\chi_{\mathrm{h}_{n}}^{n} D_{\mathrm{h} n}, \\
& \frac{k_{\mathrm{h} n}^{2}-\kappa_{\mathrm{h}}^{2}}{k_{\mathrm{h} n}^{2}} D_{\mathrm{h} n}=\chi_{\mathrm{h}_{n}^{n}}^{n} D_{0 n}+\chi_{0}^{n} D_{\mathrm{h} n},
\end{aligned}
$$

where $\kappa_{0}$ and $\kappa_{\mathrm{h}}$ are the values of the incident and diffracted wave vectors in vacuum, respectively (see Fig. 1).

The lateral components of all vectors $\boldsymbol{k}_{0 n}$ and $\boldsymbol{k}_{\mathrm{h} n}$ coincide because they remain unchanged at refraction and specular reflection. Then, Eqs. (3) can be expressed in terms of the normal wave-vector components, which are determined by the incidence and exit angles (see Fig. 1): $\kappa_{0 \mathrm{z}}=\kappa \sin \Phi_{0}=\kappa \gamma_{0}, \quad \kappa_{\mathrm{hz}}=\kappa \sin \Phi_{\mathrm{h}}=\kappa \gamma_{\mathrm{h}} . \quad h_{\mathrm{zn}}=\kappa \psi_{n}$ $=\kappa \psi\left(1-\Delta a_{\mathrm{z}}^{n} / a\right)$, where $\psi=\boldsymbol{h} \cdot \boldsymbol{Z} / \kappa=-2 \sin \varphi \sin \theta_{B}$. Making these substitutions ${ }^{72}$ and introducing the dimensionless complex parameters $u_{n}=k_{0 \mathrm{z} n} / \kappa$ we arrive at ${ }^{17,73}$

$$
\begin{gathered}
\left(u_{n}^{2}-\gamma_{0}^{2}-\chi_{0}^{n}\right) D_{0 n}=\chi_{\overline{\mathrm{h}}_{n}}^{n} D_{\mathrm{h} n}, \\
{\left[\left(u_{n}+\psi_{n}\right)^{2}-\gamma_{\mathrm{h}}^{2}-\chi_{0}^{n}\right] D_{\mathrm{h} n}=\chi_{\mathrm{h}_{n}}^{n} D_{0 n} .}
\end{gathered}
$$

The transition from Eq. (3) to (4) makes use of the assumption that the difference between $k_{0 n}, k_{\mathrm{h} n}$, and $\kappa_{0}=\kappa_{\mathrm{h}}$ is small, so that the former two can be replaced by $\kappa$ in the denominators at the left side of Eq. (3). This is the typical approximation used in most $\mathrm{x}$-ray diffraction theories. It is valid at small deviations from the Bragg condition, $\alpha=\left(2 \boldsymbol{\kappa}_{0} \cdot \boldsymbol{h}+h^{2}\right) / \kappa^{2} \ll 1$, which is well justified for most $\mathrm{x}-$ ray diffraction experiments, since $\alpha \simeq 1$ is usually not accessed experimentally because of low reflectivity on the far tails of Bragg peaks. Thus, the range $\alpha \simeq 1$ is outside the scope of our study. For those interested in possible extensions of the theory to $\alpha \simeq 1$ (so-called asymptotic Bragg diffraction, ${ }^{74}$ or crystal truncation rod scattering ${ }^{75}$ ) the solution to Eq. (3) and the corrections to boundary conditions at large $\alpha$ were obtained by Caticha. ${ }^{76}$ In addition, as shown by Colella $^{77}$ the cases with $\alpha \simeq 1$ may require analysis in the framework of multiple Bragg diffraction theory, since the two-wave approximation (1) and (2) may become invalid.

The condition $\boldsymbol{\kappa}_{\mathrm{h}}^{2}=\boldsymbol{\kappa}_{0}^{2}$ presuming the elastic scattering of $\mathrm{X}$ rays gives ${ }^{59}$

$$
\gamma_{\mathrm{h}}^{2}=\left(\gamma_{0}+\psi\right)^{2}-\alpha,
$$

Eq. (5) implies that the exit angle of a grazing diffracted wave does not depend on vertical strains.

The values of $u_{n}$ are determined by the dispersion equation, which is the condition for the existence of a solution of Eqs. (4):

$$
\left(u_{n}^{2}-\gamma_{0}^{2}-\chi_{0}^{n}\right)\left[\left(u_{n}+\psi_{n}\right)^{2}-\gamma_{\mathrm{h}}^{2}-\chi_{0}^{n}\right]=\chi_{\mathrm{h}_{n}}^{n} \chi_{\mathrm{h}_{n}}^{n} .
$$

Equation (6) is a fourth-degree polynomial equation for $u_{n}$ and has therefore four roots. As shown in Ref. 24, there are always two roots corresponding to $\mathrm{x}$-ray waves with amplitudes damping out with $z\left[\operatorname{Im}\left(u_{n}\right)>0\right]$, and two other roots corresponding to the waves with amplitudes growing with $z$ $\left[\operatorname{Im}\left(u_{n}\right)<0\right]$. The latter waves are usually treated as being specularly reflected from the lower interfaces of the layers. We shall assume that the roots $u_{n}^{j}$ are sorted over descending $\operatorname{Im}\left(u_{n}^{j}\right)$, so that $j=3,4$ correspond to the reflected waves. For each of the solutions Eqs. (4) give $(j=1, \ldots, 4)$

$$
D_{\mathrm{h} n}^{j}=v_{n}^{j} D_{0 n}^{j}, \quad v_{n}^{j}=\left[\left(u_{n}^{j}\right)^{2}-\gamma_{0}^{2}-\chi_{0}^{n}\right] / \chi_{\overline{\mathrm{h}}_{n}}^{n} .
$$

Proceeding to the boundary conditions at multilayer interfaces, one must choose the parameters $\phi_{n}$ in a way that provides a continuous phase of the waves in Eqs. (1) and (2). This is provided by the following choice which does not affect Eqs. (3)-(7):

$$
\phi_{n}=\sum_{k=1}^{n-1} h_{\mathrm{z} k}\left(z_{k}^{L}-z_{k}^{U}\right)=\sum_{k=1}^{n-1} h_{\mathrm{zk}} t_{k},
$$

where $t_{k}$ are the thicknesses of the layers and $z_{k}^{U, L}$ denote the coordinates of the upper and lower interfaces, $z_{k}^{U}=z_{k-1}^{L}$. With the substitution of Eq. (8), the exponents in the expansions (1) and (2) become identical to $\pm \int_{0}^{z} h_{\mathrm{z}}(z) \mathrm{d} z$; they can also be presented in a more usual notation (compare with Ref. 60):

$$
\begin{aligned}
h_{\mathrm{z} n}\left(z-z_{n}^{U}\right)+\sum_{k=1}^{n-1} h_{\mathrm{z} k} t_{k} & =h_{\mathrm{z}} z+\int_{0}^{z} \Delta h_{\mathrm{z}}(z) \mathrm{d} z \\
& =h_{\mathrm{z}} z-h_{\mathrm{z}} \int_{0}^{z} \frac{\Delta a_{\mathrm{z}}(z)}{a} \mathrm{~d} z \\
& =h_{\mathrm{z}} z-h_{\mathrm{z}} u(z),
\end{aligned}
$$


where $u(z)$ is the function representing the displacement of atoms from their positions in the Takagi-Taupin theory. Equation (1) with $\phi_{n}$ given by Eq. (8) is equivalent to the expansions of the susceptibility $\chi(\boldsymbol{r})$ of strained crystals used by Takagi ${ }^{60}$ and, in fact, in the Darwin-like theory of heterostructure diffraction. ${ }^{65}$ It does not contain approximations about slow variations of $\chi(\boldsymbol{r})$ at interatomic distances. As to expansion (2), its validity will be discussed in Secs. VI-VIII.

The boundary conditions for grazing waves need to be formulated not only for x-ray amplitudes, but also for their derivatives, which corresponds to accounting for x-ray refraction and specular reflection effects. ${ }^{14,17,22}$ The transmitted and diffracted wave field of each layer are matched to those of the upper and lower adjacent layers. The wave fields in the surface layer are matched at the surface to the following vacuum x-ray field which consists of incident, specularly reflected, and diffracted waves with the amplitudes $E_{0}, E_{\mathrm{s}}$, and $E_{\mathrm{h}}$, respectively (see Fig. 1):

$$
E_{\mathrm{v}}(\boldsymbol{r})=e^{i \boldsymbol{\kappa}_{0 \|} \cdot \boldsymbol{r}}\left[E_{0} e^{i \kappa_{0} \gamma_{0} z}+E_{\mathrm{s}} e^{-i \kappa_{0} \gamma_{0} z}+E_{\mathrm{h}} e^{-i \kappa_{0} \gamma_{\mathrm{h}} z+i \boldsymbol{h}_{\|} \cdot \boldsymbol{r}}\right] .
$$

The boundary conditions provide four equations for the x-ray amplitudes at each interface, which can be formulated in the $(4 \times 4)$ matrix form: ${ }^{45,48,58,59}$

$$
\begin{gathered}
\mathcal{S}_{\mathrm{v}} \mathcal{E}_{\mathrm{v}}=\mathcal{S}_{1} \mathcal{D}_{1}, \\
\mathcal{S}_{1} \mathcal{F}_{1}^{(L)} \mathcal{D}_{1}=\mathcal{S}_{2} \mathcal{F}_{2}^{(U)} \mathcal{D}_{2}, \\
\ldots \\
\ldots, \\
\mathcal{S}_{N-1} \mathcal{F}_{N-1}^{(L)} \mathcal{D}_{N-1}=\mathcal{S}_{N} \mathcal{F}_{N}^{(U)} \mathcal{D}_{N},
\end{gathered}
$$

Here $\mathcal{E}_{\mathrm{v}}=\left(E_{0}=1,0, E_{s}, E_{h}\right)$ and $\mathcal{D}_{n}=\left(D_{0 n}^{1}, D_{0 n}^{2}, D_{0 n}^{3}, D_{0 n}^{4}\right)$ are the four-component vectors, and $\mathcal{S}_{\mathrm{v}}, \mathcal{S}_{n}$, and $\mathcal{F}_{n}$ are the characteristic $(4 \times 4)$ matrices of the layers:

$$
\mathcal{S}_{\mathrm{v}}=\left(\begin{array}{cccc}
1 & 0 & 1 & 0 \\
0 & 1 & 0 & 1 \\
\gamma_{0} & 0 & -\gamma_{0} & 0 \\
0 & \gamma_{\mathrm{h}} & 0 & -\gamma_{\mathrm{h}}
\end{array}\right), \mathcal{S}_{n}=\left(\begin{array}{cccc}
1 & 1 & 1 & 1 \\
v_{n}^{1} & v_{n}^{2} & v_{n}^{3} & v_{n}^{4} \\
u_{n}^{1} & u_{n}^{2} & u_{n}^{3} & u_{n}^{4} \\
w_{n}^{1} & w_{n}^{2} & w_{n}^{3} & w_{n}^{4}
\end{array}\right),
$$

$\left[F_{n}^{j(U, L)}\right]_{i j}=\delta_{i j} \exp \left[i u_{n}^{j} \kappa z_{n}^{(U, L)}\right]$, and $w_{n}^{j}=v_{n}^{j}\left(u_{n}^{j}+\psi_{n}\right)$.

A direct formal solution to Eqs. (11) is

$$
\mathcal{E}_{\mathrm{v}}=\mathcal{S}_{\mathrm{v}}^{-1} \mathcal{S}_{1} F_{1} \mathcal{S}_{1}^{-1} \mathcal{S}_{2} F_{2} \ldots \mathcal{S}_{N-1}^{-1} \mathcal{S}_{N} \mathcal{F}_{N}^{(U)} \mathcal{D}_{N}
$$

where $\left(F_{n}\right)_{i j}=\left[\mathcal{F}_{n}^{(U)}\left(\mathcal{F}_{n}^{(L)}\right)^{-1}\right]_{i j}=\delta_{i j} \exp \left(-i u_{n}^{j} \kappa t_{n}\right)$. After calculating the matrix product on the right hand of Eq. (13) and taking into account that the amplitudes of the waves reflected from the lower interface of a thick substrate layer are zero $\left(D_{0 N}^{3}=D_{0 N}^{4}=0\right)$, one arrives at four linear equations for four unknown amplitudes: $E_{s}, E_{s}, D_{0 N}^{1}$, and $D_{0 N}^{2}$. The other amplitudes are given by Eq. (11). This is the transfer matrix solution to the diffraction problem, as suggested in Refs. 48, 58, and 59.

\section{NUMERICAL PROBLEMS WITH THE DIRECT $(4 \times 4)$ MATRIX SOLUTION}

One of the key steps of the direct $(4 \times 4)$ matrix solution (13) is the calculation of the matrix product at the right hand of (13). This product diverges for an infinitely thick multilayer due to the accumulation of large exponents contained in $F_{k}$. In practical computations, the loss of precision in adding big numbers $\exp \left(-i u_{n}^{1,2} \kappa t_{n}\right)$ and small numbers $\exp \left(-i u_{n}^{3,4} \kappa t_{n}\right)$ happens when the multilayer thickness is greater than the $\mathrm{x}$-ray extinction depth inside a crystal. As is well known, the x-ray extinction depth at grazing angles can be as small as a few nanometers, so numerical problems may arise for quite thin structures.

A previous solution to the numerical problems was as follows. ${ }^{58,48}$ Successively calculating the matrix product in Eq. (13) from the left to the right, one is traveling from the crystal surface towards the deeper layers. If the matrix product becomes great at some layer, it indicates that the x-ray waves in this layer are very weak and the contribution to $\mathcal{E}_{\mathrm{v}}$ coming from the layer's lower interface and from all the underlying layers can be neglected. Then, the overflow is overcome since the matrix product is truncated at the upper part of the multilayer. This idea is well understood for a perfect crystal formally subdivided into multilayers. Then, $\mathcal{S}_{k}^{-1} \mathcal{S}_{k+1}=1$ and the matrix product is the inverted absorption factor of x-ray waves: $F_{1} F_{2} \ldots F_{n}=\left[\mathcal{F}_{n}^{(L)}\right]^{-1}$.

The above-described procedure is equivalent to the usual thick crystal approximation (TCA) widely used in the dynamical diffraction theory. ${ }^{69-71}$ That is, the solutions of the dispersion equation corresponding to the waves growing with $z\left[\operatorname{Im}\left(u_{n}\right)<0\right]$ are disregarded for thick crystal plate. However, as soon as the x-ray extinction strongly depends on the grazing diffraction angles, the number of layers taken into consideration may vary across a diffraction curve, so that the grazing-case TCA is dynamical. Essentially the same idea to overcome the numerical problems was suggested by Berreman and Macrander ${ }^{57}$ for their matrix differential diffraction equations of grazing-incidence diffraction.

The application of the dynamical TCA to the transfer matrix method provided a successful interpretation to the grazing-incidence diffraction measurements of strained superlattices. ${ }^{48}$ However, we have found some cases where TCA is unable to avoid numerical failures.

The problem is that the four different $\mathrm{x}$-ray wave modes $\left(D_{n}^{1}, D_{n}^{2}, D_{n}^{3}, D_{n}^{4}\right)$ are characterized by different extinction lengths inside a crystal and may set different conditions for TCA. This is clearly seen in the case of grazing-incidence diffraction [Fig. 1(a)]. In the GID conditions, the dispersion equation (6) always gives a Borrmann wave field $D_{n}^{1}$ with wave nodes between the diffraction planes and weak absorption, and an anti-Borrmann wave field $D_{n}^{2}$ with wave nodes on the diffraction planes and strong absorption. ${ }^{78,22}$ Since the Borrmann and anti-Borrmann modes are characterized by a strong and a weak interaction with crystal matter, they possess different critical angles for total external reflection, which are lower and higher, respectively, than the usual critical angle $\Phi_{\mathrm{c}}=\left(\chi_{0}\right)^{1 / 2}$. At the exact Bragg position $(\alpha=0)$ the critical angles are ${ }^{14} \Phi_{1,2}=\left(\chi_{0} \mp \chi_{\mathrm{h}}\right)^{1 / 2}$, and for the general case the angular areas for total external reflection are shown by the hatched patterns I and II in Fig. 2(a). The wave 

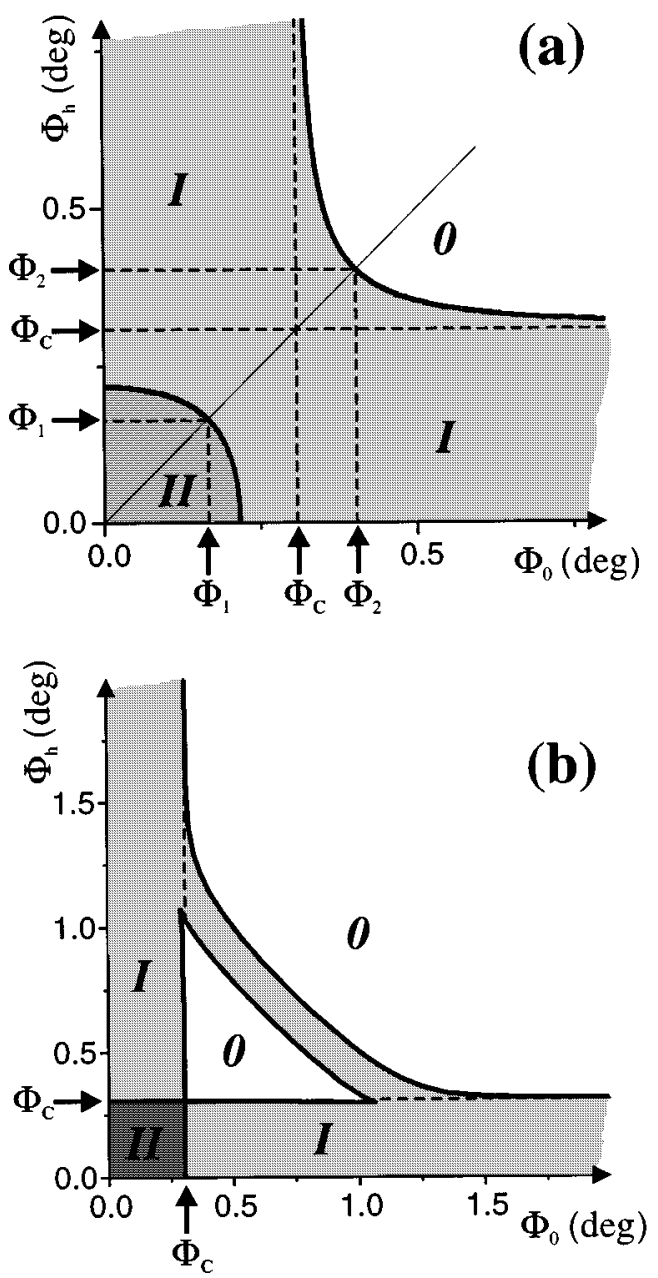

FIG. 2. The angular areas of total external reflection for (a) grazing-incidence $x$-ray diffraction and (b) grazing Bragg-Laue diffraction $\left(\varphi=-1.6^{\circ}\right)$. The calculations are for (220) reflection of $\mathrm{Cu} \mathrm{K} \alpha_{1}$ radiation from GaAs crystal. Areas denoted 0, I, and II correspond to the total reflection for none, one, and two wave fields in thick crystal, respectively. $\Phi_{0}$ is the incidence angle and $\Phi_{\mathrm{h}}$ is the exit angle of diffracted wave, $\Phi_{c}=\left(\chi_{0}\right)^{1 / 2}$ is the critical angle for total reflection in the absence of the Bragg diffraction, $\Phi_{1,2}=\left(\chi_{0} \mp \chi_{\mathrm{h}}\right)^{1 / 2}$ are the critical angles for GID introduced in Ref. 14.

fields $D_{n}^{3}$ and $D_{n}^{4}$ with $\operatorname{Im}(u)<0$ are also Borrmann and anti-Borrmann modes, and the total reflection areas for these modes coincide with that of the modes 1 and 2, respectively. The same consideration is applicable to the grazing BraggLaue diffraction [see Fig. 2(b)] and to the EAD.

The TCA procedure works well for the areas 0 and II in Fig. 2 where either none or all of the waves are strongly absorbed. In the area I (in the gap between the two critical angles) the extinction (the penetration depth) for the antiBorrmann and Borrmann modes may be of the order of $10^{2}$ $\AA$ and $10^{5} \AA$, respectively. As a result of this great difference by three orders, the anti-Borrmann mode may give large exponents in Eq. (13) and require the TCA at a few layers, while the Borrmann mode would require taking into account diffraction in the whole multilayer. One cannot use separate thick crystal approximations for different wave modes because they are coupled via the boundary conditions at the

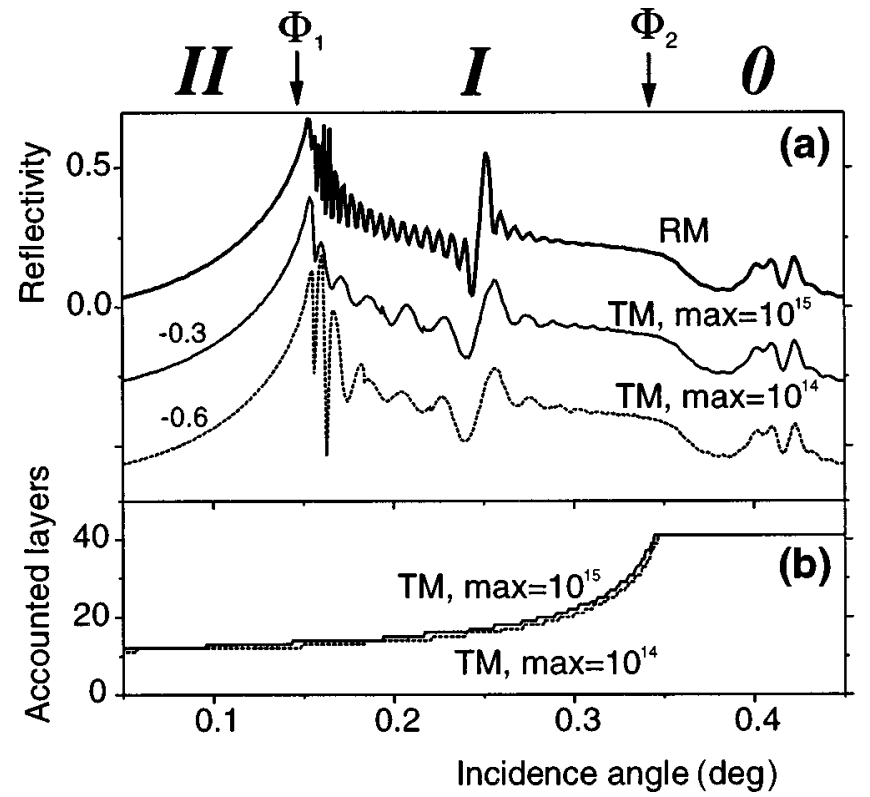

FIG. 3. An example of numerical problems that occur with the transfer matrix method. The calculations are for (220) grazingincidence diffraction of $\mathrm{Cu} \mathrm{K} \alpha_{1}$ radiation and an AlAs/GaAs superlattice [20 periods of $73 \AA \mathrm{GaAs}$ and $154 \AA$ AlAs on (001) GaAs substrate]. The scan is calculated at $\alpha=0$ and corresponds to the diagonal in Fig. 2(a). The dotted line and the thin solid line in (a) show the GID reflectivity calculated by the TM method with the $10^{14}$ and $10^{15}$ thresholds of the maximum matrix element, respectively (the curves are shifted by -0.3 for clarity). Respective lines in (b) show the number of the top layers in the multilayer taken into account in the calculations. The calculations for different thresholds disagree with each other and with the recursion matrix calculations [thick solid line in (a)] in the gap between the two critical angles for total external reflection $\left(\Phi_{1}<\Phi_{0}<\Phi_{2}\right)$.

interfaces: the anti-Borrmann waves are excited in deep layers by the Borrmann waves.

An illustration to this problem is given in Fig. 3 for (220) GID of an $\mathrm{AlAs} / \mathrm{GaAs}$ superlattice consisting of 20 periods of $73 \AA$ GaAs and $154 \AA$ AlAs on (001) GaAs substrate. The dotted and thin solid lines in Fig. 3(a) show the reflectivity curves of GID calculated by the TM method with the TCA applied when the maximum element of the matrix product is $10^{14}$ and $10^{15}$, respectively. The same lines in part (b) of the figure show how many layers out of a total of 41 are taken into account. The curves are plotted as a function of the incidence angle at $\alpha=0$. This is the scan along the diagonal in Fig. 2(a). The reflectivity curves with different TCA conditions coincide at $\Phi_{0}<\Phi_{1}$, where all the x-ray waves are totally reflected and at $\Phi_{0}>\Phi_{2}$, where nothing is totally reflected. However, they differ in the gap between the two critical angles where the anti-Borrmann waves are reflected and the Borrmann ones are not. This proves that the TCA procedure is not applicable in this range.

The TCA thresholds used in the above example are the maximum ones achievable with a double-precision FORTRAN program where the mantissa is 16 decimal digits. Performing computations with a longer mantissa may overcome the loss of precision in some cases, but cannot solve the problem in principle. 


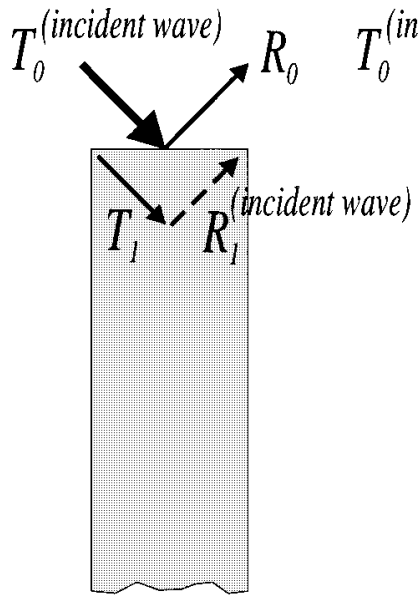

(a)

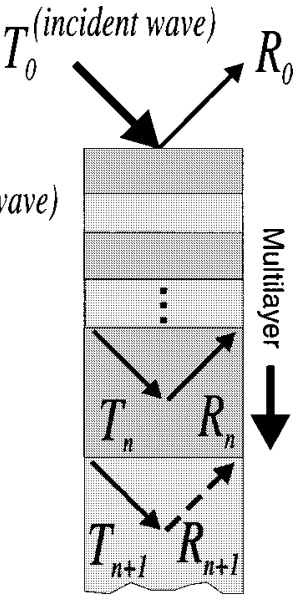

(b)
FIG. 4. On the derivation of matrix recursion equations for x-ray diffraction in cases of single heterostructure (a) and multilayer (b). $T_{k}$ and $R_{k}$ denote the two-component vectors containing the amplitudes of transmitted and reflected waves, respectively.

Thus, the matrix technique must be reformulated in order to overcome the divergences.

\section{RECURSION $(2 \times 2)$ MATRIX FORMULAS FOR MULTILAYER DIFFRACTION}

In the following consideration we make use of the approach developed by $\mathrm{Kohn}^{79}$ for nongrazing x-ray diffraction with multiple Bragg- and Laue-case $\mathrm{x}$-ray waves in multilayers. The Bragg- and Laue-case $\mathrm{x}$ rays in that problem can be viewed as being analogous to the transmitted and reflected waves in our problem. The basic idea by Kohn is that Eq. (13) diverges because the vacuum amplitudes $\mathcal{E}_{\mathrm{v}}$ are sought together with the substrate amplitudes $\mathcal{D}_{N}$. The former amplitudes are of the order of 1 , while the latter ones can be evanescent in a thick crystal. A better way is to express the reflectivity of a multilayer containing $n+1$ interfaces via that of a multilayer with $n$ interfaces. Such a recursion must converge because the effect of additional lower interfaces on the reflectivity decreases with the distance of the interfaces from the surface.

We start with the following renormalization of the x-ray amplitudes: ${ }^{80}$

$$
\mathcal{D}_{n}^{\prime}=\mathcal{F}_{n}^{(L)} \mathcal{D}_{n},
$$

and denoting $X_{n+1}=\mathcal{S}_{n}^{-1} \mathcal{S}_{n+1}$. Then, all equations (11) assume the universal form (here and below the primes in $\mathcal{D}_{n}$ are left out):

$$
\mathcal{D}_{n}=X_{n+1} F_{n+1} \mathcal{D}_{n+1}, n=0, \ldots, N-1 .
$$

The amplitudes $\mathcal{D}_{n}$ are constant within the layers and change at the interfaces. Therefore, the interfaces can be treated as "scatterers" for amplitudes. First, let us consider the scattering at a single interface. For clarity we discuss the crystal surface [Fig. 4(a)], but our consideration is applicable to any internal interface as well. The waves at the left hand of Eq. (15) can be classified as two incident and two scattered waves. We group them in the vectors $T_{0}=\left(E_{0}, 0\right)$ and
$R_{0}=\left(E_{s}, E_{h}\right)$, respectively. Also, the waves at the right hand of Eq. (15) can be viewed as two transmitted $\left[\operatorname{Im}\left(u^{1,2}\right)>0\right]$ and two incident waves $\left[\operatorname{Im}\left(u^{3,4}\right)<0\right]$. The amplitudes of the latter waves coming to the surface from the crystal interior are zero in thick crystals, but we keep them for the general case where crystals have internal interfaces. Thus, we group the waves below the surface as the vectors $T_{1}=\left(D_{0}^{1}, D_{0}^{2}\right)$ and $R_{1}=\left(D_{0}^{3}, D_{0}^{4}\right)$, respectively. Splitting matrices $X$ and $F$ into four $(2 \times 2)$ blocks we obtain

$$
\left(\begin{array}{c}
T_{0} \\
R_{0}
\end{array}\right)=\left(\begin{array}{cc}
X^{t t} & X^{t r} \\
X^{r t} & X^{r r}
\end{array}\right)\left(\begin{array}{cc}
F_{+} & 0 \\
0 & F_{-}
\end{array}\right)\left(\begin{array}{c}
T_{1} \\
R_{1}
\end{array}\right),
$$

where $F_{+}$and $F_{-}$are diagonal matrices containing the increasing and decreasing exponential functions, respectively.

Equation (16) enables the "scattered" waves $R_{0}$ and $T_{1}$ to be expressed via the "incident" waves $T_{0}$ and $R_{1}$ :

$$
\left(\begin{array}{c}
T_{1} \\
R_{0}
\end{array}\right)=\left(\begin{array}{ll}
M^{t t} & M^{t r} \\
M^{r t} & M^{r r}
\end{array}\right)\left(\begin{array}{c}
T_{0} \\
R_{1}
\end{array}\right)
$$

where

$$
\begin{gathered}
M^{t t}=\left(F^{+}\right)^{-1}\left(X^{t t}\right)^{-1}, \\
M^{t r}=-M^{t t} X^{t r} F^{-}, \\
M^{r t}=X^{r t}\left(X^{t t}\right)^{-1}, \\
M^{r r}=\left(X^{r r}-M^{r t} X^{t r}\right) F^{-} .
\end{gathered}
$$

Equations (18) have a clear physical interpretation. For example, the block $M^{r r}$ is responsible for the scattering of $R_{1}$ into $R_{0}$ and the last line in Eq. (18) implies that the scattering may be a direct transmission $R_{1} \rightarrow R_{0}$ and may be a multiple scattering process $R_{1} \rightarrow T_{0} \rightarrow T_{1} \rightarrow R_{0}$. We note that Eqs. (17) and (18) do not cause any divergences because the increasing exponentials $F^{+}$are inverted. In the case of a thick substrate vector $R_{1}$ approaches zero, and then $R_{0}=M^{r t} T_{0}$.

Proceeding to multilayers [Fig. 4(b)], the solutions of the scattering problem for multilayers incorporating $n$ interfaces and $n+1$ interfaces according to Eq. (13) can be presented as

$$
\left(\begin{array}{c}
T_{n} \\
R_{0}
\end{array}\right)=\left(\begin{array}{cc}
W_{n}^{t t} & W_{n}^{t r} \\
W_{n}^{r t} & W_{n}^{r r}
\end{array}\right)\left(\begin{array}{c}
T_{0} \\
R_{n}
\end{array}\right),
$$

and

$$
\left(\begin{array}{c}
T_{n+1} \\
R_{0}
\end{array}\right)=\left(\begin{array}{ll}
W_{n+1}^{t t} & W_{n+1}^{t r} \\
W_{n+1}^{r t} & W_{n+1}^{r r}
\end{array}\right)\left(\begin{array}{c}
T_{0} \\
R_{n+1}
\end{array}\right),
$$

respectively. Here $W_{n}$ and $W_{n+1}$ are $(2 \times 2)$ matrices. At the same time, according to Eq. (17) the scattering equations for interface $(n+1)$ are

$$
\left(\begin{array}{c}
T_{n+1} \\
R_{n}
\end{array}\right)=\left(\begin{array}{ll}
M_{n+1}^{t t} & M_{n+1}^{t r} \\
M_{n+1}^{r t} & M_{n+1}^{r r}
\end{array}\right)\left(\begin{array}{c}
T_{n} \\
R_{n+1}
\end{array}\right) .
$$

The combination of Eqs. (19)-(21) results in the following recursion formulas for $W_{n}$ : 


$$
\begin{gathered}
W_{n+1}^{t t}=A_{n} W_{n}^{t t}, \\
W_{n+1}^{t r}=M_{n+1}^{t r}+A_{n} W_{n}^{t r} M_{n+1}^{r r}, \\
W_{n+1}^{r t}=W_{n}^{r t}+B_{n} M_{n+1}^{r t} W_{n}^{t t}, \\
W_{n+1}^{r r}=B_{n} M_{n+1}^{r r},
\end{gathered}
$$

where it is denoted

$$
\begin{aligned}
& A_{n}=M_{n+1}^{t t}\left(1-W_{n}^{t r} M_{n+1}^{r t}\right)^{-1}, \\
& B_{n}=W_{n}^{r r}\left(1-M_{n+1}^{r t} W_{n}^{t r}\right)^{-1} .
\end{aligned}
$$

Starting with the crystal surface and progressively applying Eqs. (22) to lower interfaces, one arrives at the matrices $W_{N}^{x y}$ determining the reflectivity of the whole multilayer. The recursion matrix (RM) solution does not cause any divergences in the numerical calculations. As follows from Eq. (18), the order of $M^{r t}$ is about 1, while the other three blocks are small due to the factors $F^{-}$and $\left(F^{+}\right)^{-1}$. According to Eq. (22), the same ratio of orders is preserved for the blocks $W^{x y}$. Thus, the block $W_{N}^{r t}$ is the only one significant for a thick multilayer and the solution to the diffraction problem is $R_{0}=W_{N}^{r t} T_{0}$. The other blocks converge to zero at the recursions (22).

The thick solid line on Fig. 3(a) shows the GID reflectivity calculated by the RM method for the example discussed in the previous section. The RM calculation coincides with the transfer matrix results in angular areas II and 0 . In area I, there is a disagreement, because the TM method fails. However, when the TCA threshold in the transfer matrix calculations is increased, the mismatch between the two methods decreases. A complete coincidence would be achieved if one had a computer with a hypothetically unlimited number of significant digits.

Finally, let us find the x-ray wave-field amplitudes $R_{n}$ and $T_{n}$ inside the layers. These are required for the interpretation of x-ray standing waves ${ }^{81}$ and diffuse scattering ${ }^{51}$ in diffraction from multilayers. Equation (19) gives $R_{0}=W_{n}^{r t} T_{0}+W_{n}^{r r} R_{k}$. However, the direct solution $R_{k}=\left(W_{n}^{r r}\right)^{-1}\left(R_{0}-W_{n}^{r t} T_{0}\right)$ leads to uncertainties like $0 / 0$ for thick multilayers and one has to make use of recursions. A combination of Eqs. (19) and (21) brings

$$
\begin{gathered}
R_{n}=\left(1-M_{n+1}^{r t} W_{n}^{t r}\right)^{-1}\left(M_{n+1}^{r r} R_{n+1}+M_{n+1}^{r t} W_{n}^{t t} T_{0}\right), \\
T_{n}=W_{n}^{t t} T_{0}+W_{n}^{t r} R_{n} .
\end{gathered}
$$

Equations (24) must be progressively applied to all the layers starting at the crystal substrate where $R_{N}=0$.

\section{REDUCTION TO SCALAR RECURSIONS IN PARTICULAR CASES}

\section{A. Reduction to Parratt's formulas far from the Bragg diffraction}

When the grazing $\mathrm{x}$ rays are far away from the Bragg conditions, the x-ray wave field above the surface is reduced to the incident $E_{0}$ and specular $E_{\mathrm{s}}$ waves only, and the field in each layer consists of one transmitted $D_{n}^{1}$ and one reflected $D_{n}^{2}$ wave with the wave vectors $\boldsymbol{k}_{n}^{1,2}=\left( \pm \kappa u_{n}, \boldsymbol{\kappa}_{\|}\right)$, respectively. Here $\pm u_{n}= \pm\left(\gamma_{0}^{2}+\chi_{0}^{n}\right)^{1 / 2}$ are the solutions to the dis- persion equation, the order of which is reduced to 2. The boundary conditions Eq. (11) and Eq. (13) formally remain in the same form, but all the matrices are now $(2 \times 2)$. In particular, the scattering matrices $\mathcal{S}_{\mathrm{v}}$ and $\mathcal{S}_{n}$ are reduced to

$$
\mathcal{S}_{\mathrm{v}}=\left(\begin{array}{cc}
1 & 1 \\
\gamma_{0} & -\gamma_{0}
\end{array}\right), \quad \mathcal{S}_{n}=\left(\begin{array}{cc}
1 & 1 \\
u_{n}^{1} & u_{n}^{2}
\end{array}\right)
$$

and for $X_{n+1}$ and $M_{n+1}^{x y}$ we find

$$
X_{n+1}=\left(\begin{array}{ll}
a_{n, n+1}^{+} & a_{n, n+1}^{-} \\
a_{n, n+1}^{-} & a_{n, n+1}^{+}
\end{array}\right),
$$

$$
\begin{gathered}
M_{n+1}^{t t}=\tau_{n, n+1} \exp \left(-i u_{n+1} \kappa t_{n+1}\right), \\
M_{n+1}^{t r}=r_{n+1, n} \exp \left(-2 i u_{n+1} \kappa t_{n+1}\right), \\
M_{n+1}^{r t}=r_{n, n+1}, \\
M_{n+1}^{r r}=\tau_{n+1, n} \exp \left(-i u_{n+1} \kappa t_{n+1}\right) .
\end{gathered}
$$

Here $a_{n, n+1}^{ \pm}=\left(u_{n} \pm u_{n+1}\right) / 2 u_{n}$. The parameters $\tau_{n, n+1}$ $=2 u_{n} /\left(u_{n}+u_{n+1}\right)$ and $r_{n, n+1}=\left(u_{n}-u_{n+1}\right) /\left(u_{n}+u_{n+1}\right)$ are the Fresnel transmission and reflection coefficients, respectively, for the wave incident on the interface from layer $n ; \tau_{n+1, n}$ and $r_{n+1, n}$ are those for the wave incident on the interface from layer $n+1$.

Thus, for specular reflection the recursion formulas (22) become scalar, but they do not have exactly the same form as Parratt's recursion formulas. ${ }^{63}$ The difference is that our equations express the reflectivity of a multilayer consisting of $n+1$ interfaces via that of $n$ interfaces and the reflectivity on $(n+1)$ th layer, while the Parratt equations connect the ratio $P_{n}=R_{n} / T_{n}$ with the respective ratio $P_{n+1}$ in the next layer. The two types of equations are equivalent and can be reduced to each other. For example, the easiest way to obtain the Parratt recursion formulas is to use Eq. (16):

$$
P_{n}=\frac{X_{n+1}^{r t}+X_{n+1}^{r r} F_{n+1}^{-}\left(F_{n+1}^{+}\right)^{-1} P_{n+1}}{X_{n+1}^{t t}+X_{n+1}^{t r} F_{n+1}^{-}\left(F_{n+1}^{+}\right)^{-1} P_{n+1}} .
$$

Substituting the explicit form of $X^{x y}$ we arrive at

$$
P_{n}=\frac{r_{n, n+1}+P_{n+1} e^{-2 i u_{n+1} \kappa t_{n+1}}}{1+r_{n, n+1} P_{n+1} \exp \left(-2 i u_{n+1} \kappa t_{n+1}\right)},
$$

where $r_{n, n+1}$ is the Fresnel reflectivity defined above. Equation (29) is the same as the Parratt recursion equation with the only difference that we define $P_{n}=R_{n} / T_{n}$ at the lower layer interface, while Parratt used the definition at the middle of layers. In the general case where $R_{n}$ and $T_{n}$ are not scalars, the Parratt method is not applicable, while the recursion equations (22) remain valid.

\section{B. Reduction to Bartels' formulas for nongrazing Bragg diffraction}

When $\mathrm{x}$ rays satisfy the Bragg condition and the incident and exit angles are not small, one can neglect the specular $\mathrm{X}$-ray waves. Then, only those solutions $u_{n}^{j}$ to the dispersion equation (6) are significant, for which the waves inside crystal only slightly deviate from the waves in vacuum: $u_{n}^{j} \approx \gamma_{0}$, 
or $u_{n}^{j}+\psi_{n} \approx-\gamma_{\mathrm{h}}$. After discarding unimportant roots and proceeding from the large parameters $u_{n}$ to the small refraction corrections $s_{n}=u_{n}-\left(\gamma_{0}^{2}+\chi_{0}^{n}\right)^{1 / 2}$ in Bragg diffraction, Eq. (6) is reduced to the following second-degree polynomial: $^{.25}$

$$
s_{n}\left(s_{n}-\frac{\widetilde{\alpha}_{n}}{2 \gamma_{0}}\right)+\frac{\chi_{\overline{\mathrm{h}}_{n}}^{n} \chi_{\mathrm{h}_{n}}^{n}}{4 \gamma_{0} \gamma_{\mathrm{h}}}=0 .
$$

Here $\widetilde{\alpha}_{n}=\alpha-\left(\chi_{0}^{n}+2 \gamma_{0} \gamma_{\mathrm{h}} \Delta a_{\mathrm{z}}^{n} / a\right)(1+\beta) / \beta$ is the parameter determining the deviation of $\mathrm{x}$-rays from the Bragg condition in layer $n$, and $\beta=\gamma_{0} / \gamma_{\mathrm{h}}$ is the asymmetry factor of the reflection. The roots of Eq. (30) are the well-known solutions of the dynamical diffraction theory:

$$
\begin{gathered}
s_{n}=\frac{\left(\chi_{\overline{\mathrm{h}}_{n}}^{n} \chi_{\mathrm{h}_{n}}^{n}\right)^{1 / 2}}{2\left(\gamma_{0} \gamma_{\mathrm{h}}\right)^{1 / 2}}\left(-y_{n} \pm \sqrt{y_{n}^{2}-1}\right), \\
y_{n}=-\frac{\widetilde{\alpha}_{n} \beta^{1 / 2}}{2\left(\chi_{\overline{\mathrm{h}}_{n}}^{n} \chi_{\mathrm{h}_{n}}^{n}\right)^{1 / 2}} .
\end{gathered}
$$

As soon as the refraction and specular reflection effects are small, the boundary conditions can be formulated for $\mathrm{x}$-ray wave amplitudes only, and the solution to the multilayer diffraction problem is obtained in the general form (13) with $(2 \times 2)$ scattering matrices. In this case the explicit form of $\mathcal{S}_{\mathrm{v}}$ and $\mathcal{S}_{n}$ is

$$
\mathcal{S}_{\mathrm{v}}=\left(\begin{array}{ll}
1 & 0 \\
0 & 1
\end{array}\right), \quad \mathcal{S}_{n}=\left(\begin{array}{cc}
1 & 1 \\
v_{n}^{1} & v_{n}^{2}
\end{array}\right),
$$

and the calculation of $X_{n+1}$ and $M_{n+1}^{x y}$ is straightforward, but tedious.

The important practical result is that the recursion formulas (22) become scalar. Again, as in the case of the specular reflection problem, our recursion formulas differ from that by Bartels, Hornstra, and Lobeek, ${ }^{64}$ who used the recursions for $P_{n}=R_{n} / T_{n}$. However, the two types of equations are equivalent and the formulas by Bartels, Hornstra, and Lobeek can be obtained using Eq. (28).

In extremely asymmetric Bragg diffraction where only one $\mathrm{x}$-ray wave is grazing, the dispersion equation (6) can be reduced to a third-order polynomial with three roots. ${ }^{59} \mathrm{How}-$ ever, some of the matrices in Eq. (22) then become $(2 \times 1)$ rectangular. The way to handle rectangular matrices in recursion formulas was discussed by Kohn, ${ }^{79}$ who solved this problem for multiple Bragg diffraction with no grazing $\mathrm{x}$ rays in multilayers.

\section{EXPERIMENT}

Ewald's expansion (2), the starting point of our method, is obviously valid for thick layers (mathematically-for an infinite crystal), while for thin layers composed of a few atomic planes, a continuous expansion of $D_{n}(\boldsymbol{r})$ in a Fourier integral over $\boldsymbol{h}$ may be required. Thus one has to prove that using the expansions over the local periodicity of atomic planes gives results that are consistent with experiment and with other theories containing no such assumption.
In order to give an experimental verification to our theory, we have carried out symmetric and extremely asymmetric Bragg diffraction measurements of an AlAs/GaAs superlattice. The sample was a 20-period AlAs/GaAs superlattice grown on a (100) GaAs substrate by molecular-beam epitaxy. The thickness of the layers was $154 \AA$ AlAs and $73 \AA$ GaAs, and the interface roughness was $4 \AA$, as found by fitting grazing-incidence $\mathrm{x}$-ray specular reflection data of the sample. The multilayer thickness was far below the critical thickness for the strain relaxation and formation of misfit dislocations. The absence of relaxation was confirmed by the measurements of symmetric 400 Bragg reflection (see below) and asymmetric x-ray topographs (the latter are not shown here). Also, no noticeable sample curvature was found.

The measurements of the superlattice can provide a good test for the assumption concerning the periodicity of $\mathrm{x}$-ray waves. First, the layers in the SL are as thin as a few monolayers and, second, a possible deviation of the real wave field from the theory will be accumulated in a resonant way at the SL peaks.

The symmetric 400 Bragg diffraction measurements were taken in the laboratory using a Philips materials research diffractometer (MRD) and $\mathrm{CuK}_{\alpha 1}$ radiation from a $2 \mathrm{~kW}$ X-ray tube monochromatized by a Ge (220) Bartels-type monochromator. The extremely asymmetric diffraction experiment was carried out at the CEMO beamline of HASYLAB, DESY. An $(n,-n)$ nondispersive setup was used with a Ge double-crystal monochromator (symmetric 311 reflection) and coplanar asymmetric 311 reflection from the sample. The asymmetry of the sample Bragg reflection was varied by changing the x-ray energy around $8.5 \mathrm{keV}$.

All the data were simulated with the help of the theory presented in Sec. IV. The experimental angles are introduced into the theory as follows: ${ }^{59}$ let $\boldsymbol{a}$ be a unit vector along the crystal scan axis. When the crystal is rotated round $\boldsymbol{a}$ through an angle $\delta \theta$, the original wave vector $\boldsymbol{\kappa}_{0}^{\mathrm{B}}$ satisfying the exact Bragg condition $(\alpha=0)$ is changed by a vector $\delta \boldsymbol{\kappa}$, which can be expanded into the two mutually perpendicular vectors $\boldsymbol{c}$ and $\boldsymbol{b}$, both lying in the plane of rotation:

$$
\begin{gathered}
\delta \boldsymbol{\kappa}=x \boldsymbol{c}+y \boldsymbol{b}, \\
\boldsymbol{b}=\boldsymbol{\kappa}_{0}^{\mathrm{B}}-\left(\boldsymbol{\kappa}_{0}^{\mathrm{B}} \cdot \boldsymbol{a}\right) \boldsymbol{a}, \quad \boldsymbol{c}=\left[\boldsymbol{\kappa}_{0}^{\mathrm{B}} \times \boldsymbol{a}\right] .
\end{gathered}
$$

Then, the conditions: $\delta \kappa=2 b \sin (\delta \theta / 2)$ and $\left(\boldsymbol{\kappa}_{0}^{\mathrm{B}}+\delta \boldsymbol{\kappa}\right)^{2}=\kappa^{2}$ give

$$
x=(b / c) \sin (\delta \theta), \quad y=-2 \sin ^{2}(\delta \theta / 2) .
$$

As soon as $\delta \boldsymbol{\kappa}$ is found, we can calculate $\alpha=2(\delta \boldsymbol{\kappa} \cdot \boldsymbol{h}) / \boldsymbol{\kappa}^{2}$ and $\gamma_{0}=\gamma_{0}^{\mathrm{B}}+(\delta \boldsymbol{\kappa} \cdot \boldsymbol{Z}) / \kappa$. The value of $\gamma_{\mathrm{h}}$ is given by Eq. (5). Unlike usual Bragg diffraction, accounting for the variations in $\gamma_{0}$ and $\gamma_{\mathrm{h}}$ during scans is absolutely necessary in grazing geometries because these parameters may change significantly.

For coplanar geometries $\left(\boldsymbol{a} \simeq\left[\boldsymbol{\kappa}_{0}^{\mathrm{B}} \times \boldsymbol{h}\right]\right)$ and small scan angles $(\delta \theta \ll 1) \quad$ Eq. (34) is simplified to $\delta \boldsymbol{\kappa} \approx \delta \theta\left[\boldsymbol{\kappa}_{0}^{\mathrm{B}} \times\left[\boldsymbol{\kappa}_{0}^{\mathrm{B}} \times \boldsymbol{h}\right]\right] /\left(\boldsymbol{\kappa} h \cos \theta_{\mathrm{B}}\right)$, which brings the wellknown expression for $\alpha=-2 \sin \left(2 \theta_{\mathrm{B}}\right) \delta \theta$.

The data for the symmetric reflection are presented in Fig. 5. The experiment and the theory are shown by dotted and 


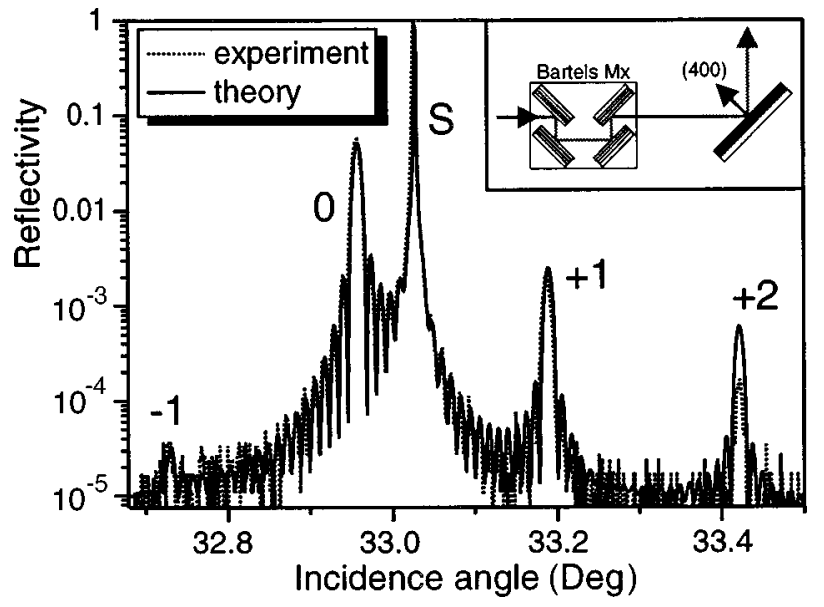

FIG. 5. A comparison of the recursion matrix theory with experiment for 400 symmetric reflection of an AlAs/GaAs superlattice. $S$ and the numbers $0,+1, \ldots$ mark the substrate Bragg peak and the different-order superlattice peaks, respectively. The inset shows the experimental setup with the (220) Bartels monochromator.

solid lines, respectively. The theoretical curve is calculated using the normal lattice spacing mismatch $(\Delta a / a)_{\mathrm{AlAs}}=2.775 \times 10^{-3}$ in the AlAs layers, as measured in the precise experiment by Bocchi et al. ${ }^{82}$ for fully strained AlAs on GaAs. The general match between the theory and the experiment is good, although the theory overestimates the reflectivity of the +2 SL peak. This can be explained by the effect of $4 \AA$ interface roughness (or by the presence of transition layers). ${ }^{83}$

In the symmetric case the RM calculations perfectly coincided with the calculations provided by the commercial Philips software based on the Bartels, Hornstra, and Lobeek algorithm. ${ }^{64}$ This fact is not surprising because the Bartels recursion formulas are a particular case of the RM method.

Figure 6 presents the experiment (dotted lines) and the theory (solid lines) for the 311 coplanar extremely asymmetric Bragg reflection from the same sample. The successive curves correspond to increasing asymmetry of the Bragg reflection which is determined by the difference $\theta_{\mathrm{B}}-\theta_{\text {misc }}$ between the kinematical Bragg angle and the miscut of the (311) planes. The actual difference deviates from this value because of the refraction effects for incident $x$ rays. The reflection asymmetry was altered by small tilts of the doublecrystal monochromator that caused small changes in the energy of incident synchrotron radiation. The upper and the lower three energies correspond to the kinematical Bragg condition above and below the total-reflection critical angle for the incident $x$ rays, respectively. In the latter case the extinction length of $\mathrm{x}$ rays decreases and the reflectivity at the substrate peak $S$ falls.

The theoretical curves are corrected for a geometrical factor (the part of the diffracted intensity measured by the detector was proportional to the incidence angle because of the large footprint of the incident $\mathrm{x}$-ray beam at the sample surface) and added to the experimental background.

As we see, the same theory with the same structure parameters explains both the symmetric and asymmetric $\mathrm{x}$-ray diffraction experiments. It should be noted that the applicability of Ewald's expansion (2) to asymmetric diffraction is justified even better than for symmetric diffraction, because

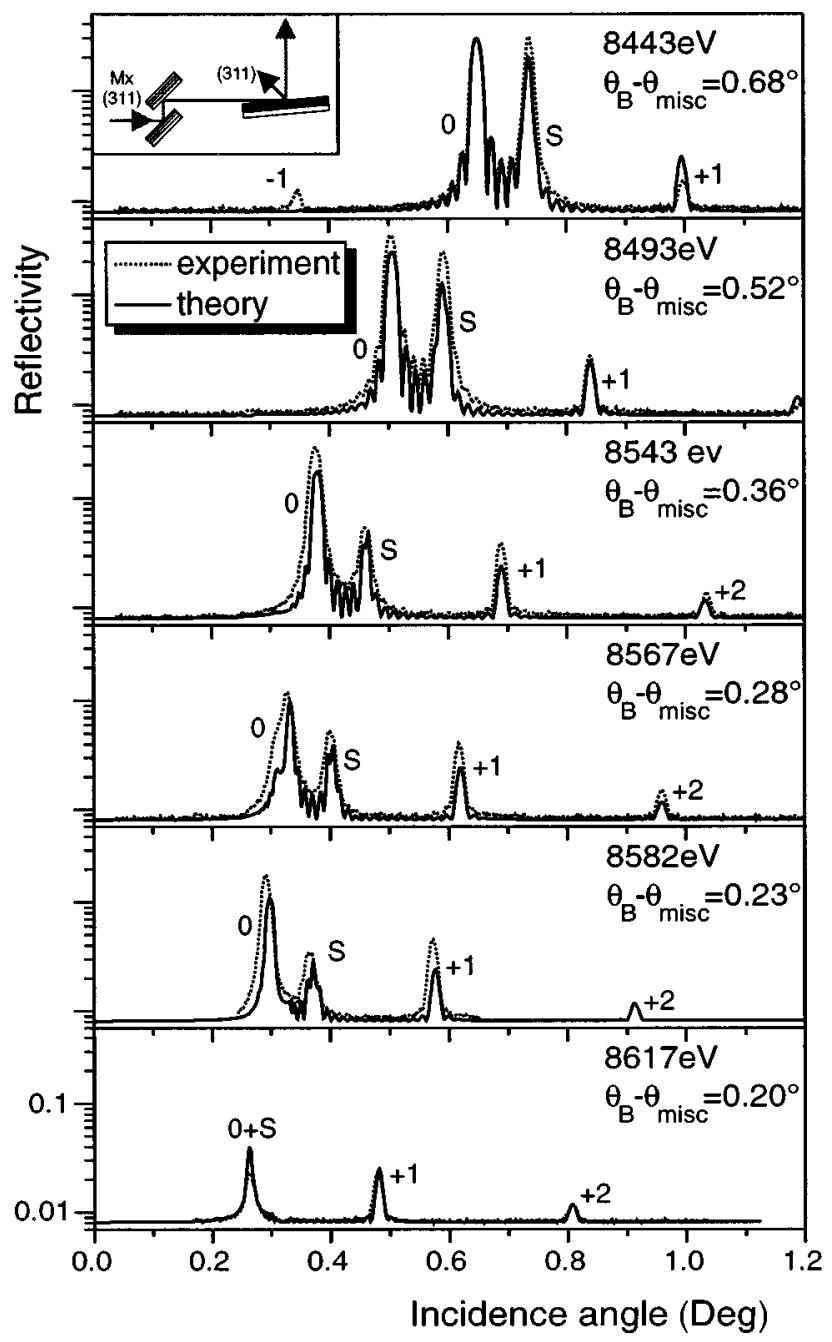

FIG. 6. Same as in Fig. 5 for 311 extremely asymmetric reflection. The insert shows the experimental setup with the doublecrystal monochromator in the nondispersive 311 Bragg position. The captions above each rocking curve indicate the asymmetry of the reflection at the respective wavelength of synchrotron radiation selected by the monochromator.

grazing $\mathrm{x}$ rays interact with a greater number of atomic planes projected on their path, which results in a shorter extinction depth.

However, the general match between the theory and the experiment in the 311 case is worse than that in the 400 case. It cannot be due to the larger angular range of this scan, because $|\alpha| \leq 0.02$, so the approximation used in Eq. (4) is well justified. Possible explanations for the mismatch can be a greater footprint of incident beam at the sample surface in asymmetric diffraction (the reflectivity is averaged over a greater surface area), or a greater sensitivity of EAD to surface defects due to a smaller extinction length. Also, it might be due to the sensitivity of EAD to the fluctuations of material density and interface roughness, ${ }^{51}$ which affect the refraction of grazing $\mathrm{x}$ rays.

We have found that an additional source of mismatch are dispersion effects in the $(n,-n)$ scheme applied to EAD. The $(n,-n)$ scheme is dispersion free for conventional geometries, because the Bragg curves for different wavelengths possess the same shape and they are simultaneously mea- 


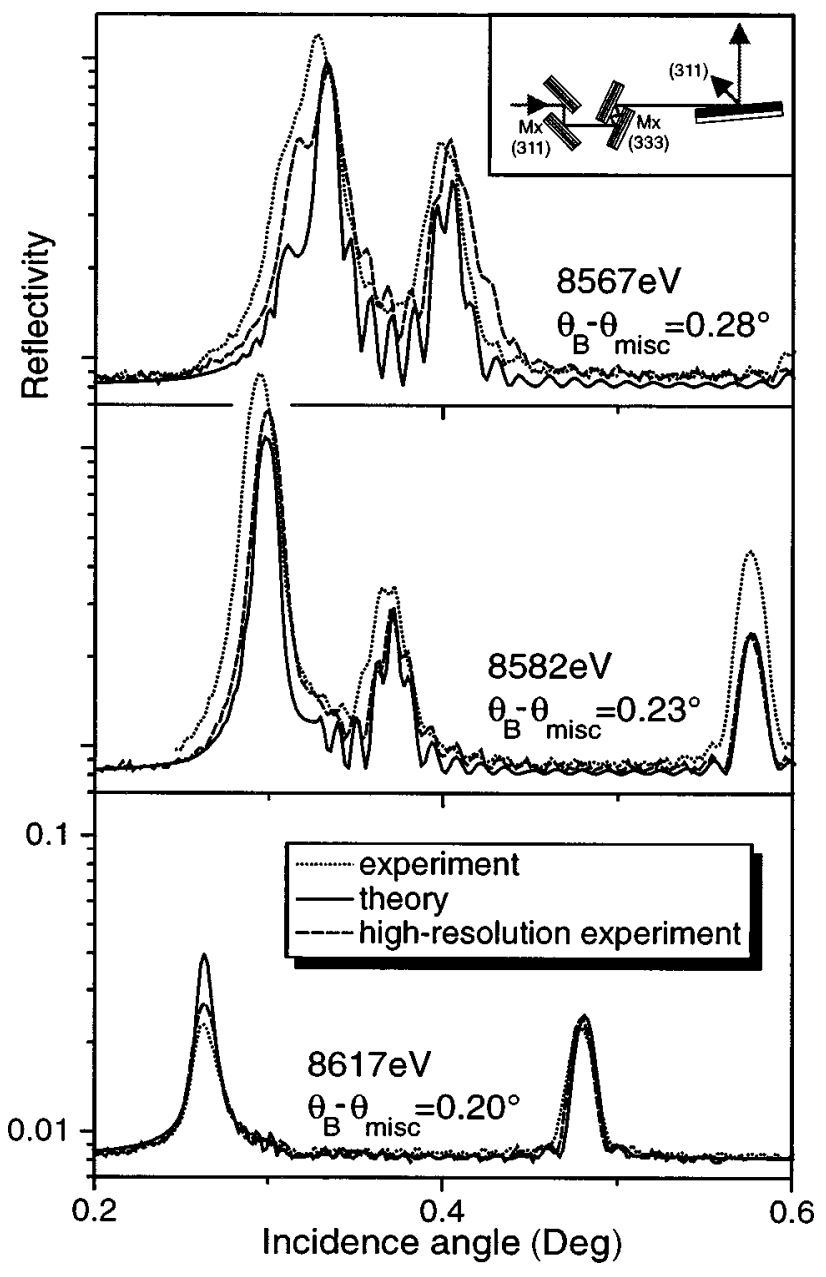

FIG. 7. Same as in Fig. 6 for the experimental curves measured with an additional (333) double-reflection monochromator reducing the wavelength dispersion.

sured at the same deviations from the Bragg angle. Thus, dispersion effects in two crystals cancel each other. This does not remain true for extremely asymmetric diffraction. One can see in Fig. 6 that when the incident energy is changed by a small value $\Delta E / E \simeq 10^{-3}$, the curves of asymmetric diffraction not only shift by the Bragg angle, but also considerably change their shape. In our case the shape of the Bragg curves depends on the angular distance between the Bragg angle and the critical angle for total external reflection. Therefore, the shape of the curves for different wavelengths is averaged in $(n,-n)$ measurements (the effect is proportional to the wavelength spread of incident $\mathrm{x}$ rays).

In order to avoid the dispersion effect, we have carried out an asymmetric diffraction experiment with an additional four-reflection $\mathrm{Si}$ (333) monochromator selecting a narrow wavelength interval. The results are presented on Fig. 7. Clearly, the experiment now tends to be in much better agreement with the theory.

Thus, we have shown that our theory gives a good explanation for both symmetric and extremely asymmetric Bragg reflections from a short-period superlattice containing 13 and 27 atomic layers of GaAs and AlAs, respectively. However, further experiments, especially with thinner layers and x-ray standing waves measurements are welcome. These could be $\mathrm{x}$-ray fluorescence measurements of interface-located atoms

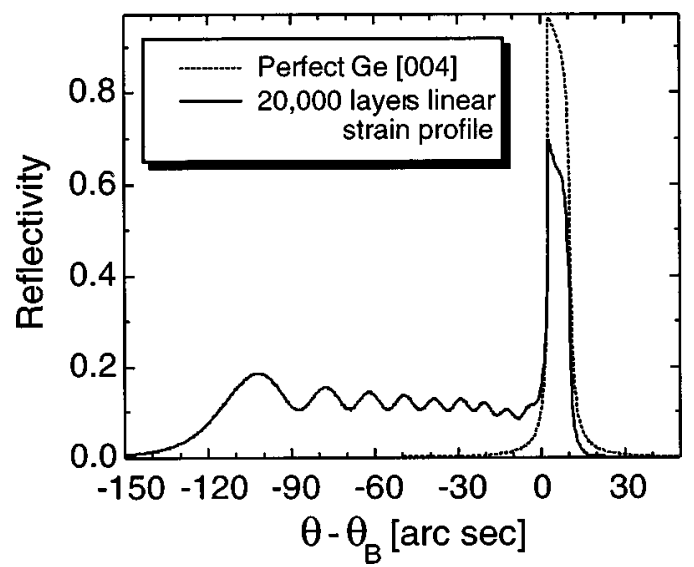

FIG. 8. Rocking curves of the 400 symmetric reflection for ideal $\mathrm{Ge}$ crystal and Ge crystal with linearly strained surface layer $\left[t=20000 a_{0}, a_{n}=(1+n \delta) a_{0}\right.$, where $n=1, \ldots 20000, \delta=5 \times 10^{-8}$, $a_{0}=1.44 \AA$ is the interplanar spacing for (400) in Ge]. The results of the calculations completely coincide with that calculated by the Darwin method and presented in Ref. 65.

under Bragg diffraction, which might provide direct information on the structure of $\mathrm{x}$-ray standing waves (XSW). Recent XSW studies of short-period (AlAs) ${ }_{3} /(\mathrm{GaAs})_{7}$ superlattices ${ }^{81}$ constitute a step in this direction, but the fluorescence yield was not interface specific.

\section{COMPARISON WITH THE DARWIN THEORY OF HETEROSTRUCTURE DIFFRACTION}

Since the assumption of the applicability of Ewald's expansion to thin layers is the most critical point of our theory, it is important to compare our results with the Darwin-type theory of heterostructure diffraction, ${ }^{65}$ which contains no assumptions of that kind, but instead directly sums up $\mathrm{x}$ rays scattered from atoms in the individual atomic planes.

Figure 8 presents the results of the RM method for an ideal example discussed by Durbin and Follis. ${ }^{65}$ The reflectivity of symmetric 400 Bragg diffraction is calculated for a hypothetical structure where a Ge crystal has a surface layer with a linearly increasing lattice parameter. The layer consists of 20000 atomic planes whose spacing $a_{n}$ successively increases towards the surface as $a_{n}=(1+n \delta) a_{0}$, where $n=1, \ldots 20000, \delta=5 \times 10^{-8}$, and $a_{0}=1.44 \AA$ is the interplanar spacing for (400) planes in Ge. In order to apply our method, we formally subdivided the strained layer into 20000 sublayers and solved the dynamical diffraction problem in each of them. Our result exactly coincides with the Darwin theory calculations, even though one cannot consider any periodicity at all in a layer consisting of just one atomic plane.

The coincidence of the two theories can be understood by observing that the dynamical diffraction solution automatically reduces to the kinematical one for a very thin layer. ${ }^{71}$ Our method thus gives the kinematical scattering of each plane, and sums up the multiple scattering exactly in the same way as in the Darwin theory.

Retracing the calculation of superlattice diffraction discussed in the experimental section, one can subdivide each layer in the SL into sublayers corresponding to the atomic planes and apply both the Darwin and our theory. The result 
of our method does not change if the perfect layers are formally subdivided into monolayers. On the other hand, it coincides with the Darwin theory if the sublayers are atomic planes. Thus, our method gives the same reflectivity as the Darwin method with the advantage that with our approach the scattering from thick layers is summed up analytically.

The above comparison is restricted by the symmetric Bragg case with ordinary incidence and exit angles. Recently it has been proposed to extend the Darwin theory to grazing incidence and/or exit by treating these cases as multiple Bragg diffraction. ${ }^{66}$ Then the Darwin theory will also require matrix recursion formulas, indicating the fundamental similarities of the two approaches.

\section{X-RAY STANDING WAVES IN MULTILAYERS}

The analysis carried out in previous sections has shown that the RM method gives correct reflectivities for x-ray diffraction of multilayers. The aim of this section is to prove the identity between x-ray standing waves in our method and other theories. The main point is to understand the seeming contradiction between x-ray waves "hooked" by atomic planes in the Ewald expansion (2) used in our method and "unhooked" x-ray standing waves reported by both the Darwin and the Takagi-Taupin theories. ${ }^{65,84}$ That is, the wave field within a given layer as described by Eq. (2) must have the periodicity of the atomic planes in that layer, yet one would not expect the total standing wave field to always follow the periodicity of the individual layers, especially when it arises primarily from substrate diffraction, for example.

Let us consider a symmetric Bragg reflection from a crystal with a strained overlayer of thickness $t$ as an example. Then, the intensity of XSW can be calculated as

$$
I(z)=\left|\sum_{j=1}^{4} e^{i \kappa u_{n}^{j} z}\left[D_{0 n}^{j}+D_{\mathrm{hn} n}^{j} e^{\left.i h_{z n}\left(z-z_{n}^{(U)}\right)+i \phi_{n}\right]}\right]\right|^{2},
$$

where one uses $\left(n=1, z_{1}^{(U)}=0, \phi_{1}=0\right)$ and $\left(n=2, z_{2}^{(U)}=t\right.$, $\left.\phi_{2}=h_{z 1} t\right)$ inside the layer $(z<t)$ and the substrate $(z>t)$, respectively. Here the amplitudes $D_{0 n}^{j}$ are given by Eqs. (14) and (24), and $D_{\mathrm{h} n}^{j}$ are calculated according to Eq. (7). Since $D_{0 n}^{j}$ and $D_{\mathrm{h} n}^{j}$ are constants within the layers and the phase relation between them oscillates as $1 / h_{z n}$, the XSW corresponding to each wave mode has the periodicity of local atomic planes. However, there is also an interference between several wave modes with different $u_{n}^{j}$.

Assume that we are interested in the XSW at the incidence angle corresponding to the substrate Bragg peak, and the difference in lattice spacing of substrate and overlayer is large enough to provide a splitting of their Bragg peaks. Then, solving the dispersion equation (6) for the overlayer, we obtain two roots corresponding to a weak coupling between $D_{0}^{j}$ and $D_{\mathrm{h}}^{j}$. In the first approximation, one root $u^{1} \approx \sqrt{\gamma_{0}^{2}+\chi_{0}}$ gives the pair of waves $\left(D_{0}^{1} \simeq 1, D_{\mathrm{h}}^{1} \simeq 0\right)$ being the continuation of incident wave in the layer, and the other root $u^{2} \approx-\sqrt{\gamma_{0}^{2}+\chi_{0}}-\psi_{n}$ gives the pair $\left(D_{0}^{2} \simeq 0, D_{\mathrm{h}}^{2} \simeq 1\right)$ corresponding to the continuation of the wave diffracted by the substrate. Substituting these roots in the expression for

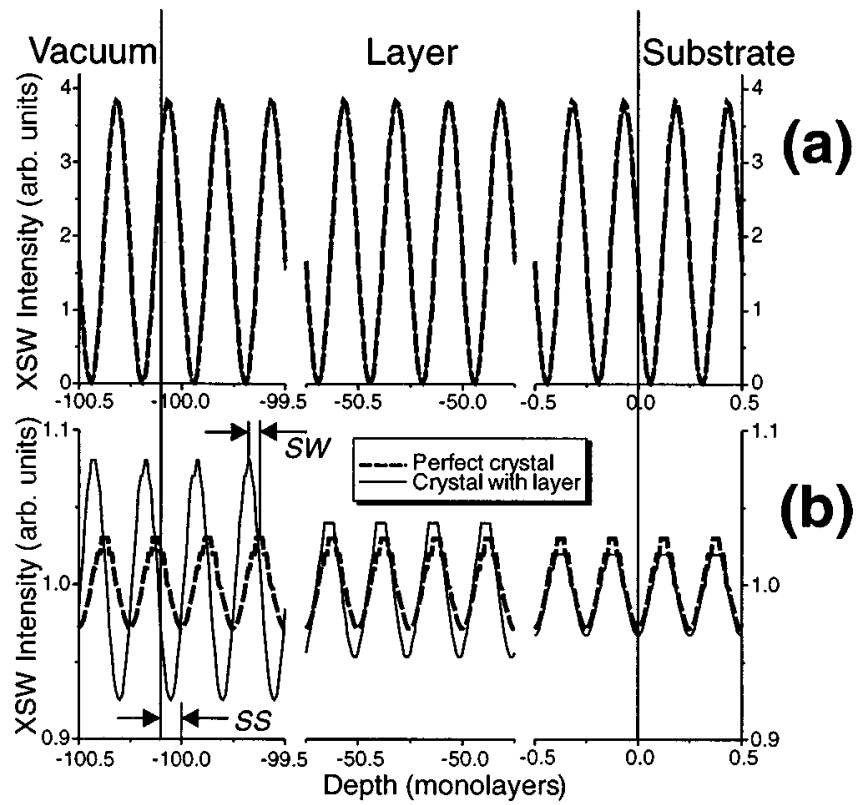

FIG. 9. The structure of x-ray standing waves at 400 symmetric Bragg reflection of $\mathrm{Cu} \mathrm{K} \alpha_{1}$ radiation from a Ge crystal with 100 surface monolayers stretched by $\Delta a / a=10^{-3}$. Solid line presents XSW for heterostructure and dashed line shows the substrate XSW extrapolated into the layer. (a) at the substrate Bragg peak; (b) at the overlayer Bragg peak. The depth is measured in Ge monolayers. SS and SW indicate the surface shift due to stretching and the XSW shift, respectively.

$\mathrm{XSW}$ in the layer:

$$
\begin{gathered}
I(z) \approx\left|e^{i \kappa u^{1} z}\left[D_{0}^{1}+D_{\mathrm{h}}^{2} e^{i P(z)}\right]\right|^{2}, \\
P(z)=\kappa\left(u^{2}-u^{1}\right) z+h_{z n}\left(z-z_{n}^{(U)}\right)+\phi_{n},
\end{gathered}
$$

we find that the term $i h_{z n} z$ is cancelled and the XSW has the periodicity of atomic planes in the substrate. However, if the deviation from the Bragg condition for the layer is small, the amplitudes $D_{\mathrm{h}}^{1}$ and $D_{0}^{2}$ become considerable and the atomic periodicity of layer may compete with that of the substrate.

To justify this conclusion, we have calculated the XSW for an overlayer consisting of 100 atomic planes on Ge (100) substrate and the symmetric 400 reflection of $\mathrm{CuK}_{\alpha 1}$ radiation. The overlayer was assumed to be stretched out by $\Delta a / a=10^{-3}$. Figures 9(a) and 9(b) present the calculated XSW as a function of the $z$ coordinate for the two angles $\left(\theta-\theta_{\mathrm{B}}\right)=6^{\prime \prime}$ and $\left(\theta-\theta_{\mathrm{B}}\right)=-128^{\prime \prime}$ corresponding to the substrate and the overlayer Bragg conditions, respectively ( $\theta_{\mathrm{B}}$ is the kinematical Bragg angle). At the substrate Bragg angle the XSW in the overlayer completely conforms with the XSW in the substrate, while at the overlayer Bragg condition the XSW starts with conformality near the substrate, and then it gradually shifts towards the expanded crystal lattice of the overlayer. Near the surface the shift with respect to the XSW extended from the substrate is as large as about $1 / 4$ of the XSW period. However, we note that in the latter case the relative intensity of the XSW is rather small. Stronger XSW correspond to thicker layers, but then the effect of "hooking" becomes evident. 
In summary, there is good agreement between our method and the results of other theories concerning $\mathrm{x}$-ray standing waves.

\section{CONCLUSIONS}

We have presented a recursion matrix theory and experimental results on $\mathrm{x}$-ray diffraction from strained multilayer crystals. It has been shown that the RM theory overcomes the numerical problems of the former transfer matrix method and is generally applicable for ordinary as well as grazing angles of $x$ rays. The RM method has been shown to reduce to the scalar recursion formulas by Bartels, Hornstra, and Lobeek $^{64}$ and by Parratt ${ }^{63}$ in cases of ordinary (nongrazing) $\mathrm{x}$-ray diffraction and grazing-incidence $\mathrm{x}$-ray reflection far from the Bragg conditions, respectively. The results of the Darwin theory for multilayer diffraction ${ }^{65}$ have also been reproduced, and the behavior of x-ray standing waves has been demonstrated to be in agreement with the predictions of the Darwin and the Takagi-Taupin theories.

The symmetric and extremely asymmetric Bragg diffraction experiments with strained AlAs/GaAs superlattice have confirmed the RM theory. A dispersion effect has been found in the $(n,-n)$ diffraction scheme applied to the measurements of extremely asymmetric Bragg diffraction and the necessity of an additional monochromator has been demonstrated in order to suppress this effect.

Extensions of the theory to relaxed multilayers containing misfit dislocations and lateral strains can be considered. The relaxation is usually characterized by a considerable difference in lateral lattice parameters, so that the Bragg peaks from different layers are well resolved and can be treated independently. Then, in the first approximation, the RM theory can be applied independently for each of the Bragg peaks with the assumption of uniformly strained layers. ${ }^{85}$ Then, scattering from strains around dislocations and other defects can be calculated as a perturbation ${ }^{86}$ using the wave fields given by the RM theory as a basis for the distorted wave Born approximation (DWBA).

The combination of the wave fields provided by the RM method and the DWBA can also be applied to the scattering from surface gratings, like, e.g., in the recent analysis of roughness effects on GID. ${ }^{51}$ The same approach can be used as well for the scattering from point defects.

Finally, some grazing-incidence $\mathrm{x}$-ray standing waves experiments from strained multilayers are in preparation, and will be useful tests of the theory.

The results of this study are aimed at stimulating the application of x-ray diffraction schemes with grazing incidence and/or exit to semiconductor structure research and surface science.

\section{ACKNOWLEDGMENTS}

This work was supported by the Volkswagen Foundation, Federal Republic of Germany (Project No 1/72439). One of us (S.A.S.) is pleased to thank T. Jach (National Institute of Standards, Gaithersburg), V. Kaganer (Institute of Crystallography, Moscow), V. Kohn (Kurchatov Institute, Moscow), A. Macrander, and S. Sinha (Argonne National Laboratory) for stimulating discussions. We are grateful to R. Hey (Paul-Drude Institute, Berlin) for the preparation of AlAs/ GaAs superlattice, and to M. Schmidbauer (Humboldt University of Berlin) for providing the calculations with the Philips software.
*Permanent address: Institute for Nuclear Problems, 11 Bobruiskaya St., Minsk 220050, Republic of Belarus.

${ }^{1}$ R. Feidenhans'l, Surf. Sci. Rep. 10, 105 (1989).

${ }^{2}$ A. M. Afanas'ev, R. M. Imamov, and E. K. Mukhamedzhanov, Cryst. Rev. 3, 157 (1992).

${ }^{3} \mathrm{H}$. Dosch, in Critical Phenomena at Surfaces and Interfaces (Evanescent X-Ray and Neutron Scattering), edited by G. Höhler, Springer Tracts in Modern Physics, Vol. 126 (SpringerVerlag, Berlin, 1992).

${ }^{4}$ S. Dietrich and A. Haase, Phys. Rep. 260, 1 (1995).

${ }^{5}$ S. Kishino and K. Kohra, J. Appl. Phys. 10, 551 (1971).

${ }^{6}$ T. Bedynska, Phys. Status Solidi A 19, 365 (1973).

${ }^{7}$ F. Rustichelli, Philos. Mag. 31, 1 (1975).

${ }^{8}$ J. Härtwig, Acta Crystallogr., Sect. A: Cryst. Phys., Diffr., Theor. Gen. Crystallogr. 37, 802 (1981).

${ }^{9}$ V. M. Kaganer, V. L. Indenbom, M. Vrana, and B. Chalupa, Phys. Status Solidi A 71, 371 (1982).

${ }^{10}$ H. R. Höche, J. Nieber, and O. Brümmer, Acta Crystallogr., Sect. A: Found. Crystallogr. 42, 585 (1986).

${ }^{11}$ A. M. Afanas'ev and O. G. Melikyan, Phys. Status Solidi A 122, 459 (1990).

${ }^{12}$ S. Kimura, J. Harada, and T. Ishikawa, Acta Crystallogr., Sect. A: Found. Crystallogr. 50, 337 (1994).

${ }^{13}$ The name "grazing incidence" is misleading in the context of this paper, since it can be applied to the other two geometries too. However, we keep it as commonly used.
${ }^{14}$ V. G. Baryshevskii, Pis'ma Zh. Tekh. Fiz. 2, 112 (1976) [Sov. Tech. Phys. Lett. 2, 43 (1976)].

${ }^{15}$ W. C. Marra, P. Eisenberger, and A. Y. Cho, J. Appl. Phys. 50, 6927 (1979).

${ }^{16}$ G. H. Vineyard, Phys. Rev. B 26, 4146 (1982).

${ }^{17}$ A. M. Afanas'ev and M. K. Melkonyan, Acta Crystallogr., Sect. A: Found. Crystallogr. 39, 297 (1983).

${ }^{18}$ A. L. Golovin, R. M. Imamov, and S. A. Stepanov, Acta Crystallogr., Sect. A: Found. Crystallogr. 40, 225 (1984).

${ }^{19}$ H. Dosch, B. W. Batterman, and D. C. Wack, Phys. Rev. Lett. 56, 1144 (1986).

${ }^{20}$ P. L. Cowan et al., Phys. Rev. Lett. 57, 2399 (1986).

${ }^{21}$ N. Bernhard et al., Z. Phys. B 69, 303 (1987).

${ }^{22}$ T. Jach, P. L. Cowan, Q. Shen, and M. J. Bedzyk, Phys. Rev. B 39, 5739 (1989).

${ }^{23}$ S. M. Durbin and T. Gog, Acta Crystallogr., Sect. A: Found. Crystallogr. 45, 132 (1989).

${ }^{24}$ P. A. Aleksandrov, A. M. Afanas'ev, and S. A. Stepanov, Kristallografiya 29, 197 (1984) [Sov. Phys. Crystallogr. 29, 119 (1984)].

${ }^{25}$ P. A. Aleksandrov, A. M. Afanas'ev, and S. A. Stepanov, Phys. Status Solidi A 86, 143 (1984).

${ }^{26}$ A. Fukuhara and Y. Takano, J. Appl. Crystallogr. 10, 287 (1977).

${ }^{27}$ B. K. Tanner and M. J. Hill, J. Phys. D 19, L229 (1986).

${ }^{28}$ L. Tapfer and K. Ploog, Phys. Rev. B 33, 5565 (1986); 40, 9802 (1989). 
${ }^{29}$ T. Kitano et al., Jpn. J. Appl. Phys., Part 2 26, L108 (1987).

${ }^{30}$ C. A. Lucas et al., J. Appl. Phys. 63, 1936 (1988).

${ }^{31}$ J. R. Buschert et al., J. Appl. Phys. 66, 3523 (1989).

${ }^{32}$ H.-G. Brühl et al., J. Appl. Crystallogr. 23, 228 (1990).

${ }^{33}$ L. Tapfer et al., Appl. Surf. Sci. 60/61, 517 (1992).

${ }^{34}$ S. Kimura, J. Mizuki, J. Matsui, and T. Ishikawa, Appl. Phys. Lett. 60, 2604 (1992).

${ }^{35}$ D. Gao, S. W. Wilkins, A. W. Stevenson, and G. N. Pain, J. Appl. Phys. 74, 3126 (1993).

${ }^{36}$ D. V. Novikov, M. Ohler, R. Köhler, and G. Materlik, J. Phys. D 28, A84 (1995).

${ }^{37}$ V. S. Wang, R. J. Matyi, and K. J. Nordheden, J. Appl. Phys. 75, 3835 (1995).

${ }^{38}$ P. A. Aleksandrov et al., J. Appl. Crystallogr. 18, 27 (1985).

${ }^{39}$ A. L. Golovin, R. M. Imamov, and E. A. Kondrashkina, Phys. Status Solidi A 88, 505 (1985).

${ }^{40}$ A. Segmüller, Thin Solid Films 154, 33 (1987).

${ }^{41}$ H. Dosch et al., Phys. Rev. Lett. 60, 2382 (1988).

${ }^{42}$ S. Grotehans et al., Phys. Rev. B 39, 8450 (1989).

${ }^{43}$ A. A. Williams et al., Phys. Rev. B 43, 5001 (1991).

${ }^{44}$ O. G. Melikyan, R. M. Imamov, and D. V. Novikov, Fiz. Tverd. Tela (Leningrad) 34, 1572 (1992) [Sov. Phys. Solid State 34, 835 (1992)].

${ }^{45}$ S. Rugel, G. Wallner, H. Metzger, and J. Peisl, J. Appl. Crystallogr. 26, 34 (1993).

${ }^{46}$ U. Pietsch et al., J. Appl. Phys. 74, 2381 (1993).

${ }^{47}$ V. H. Etgens et al., Phys. Rev. B 47, 10607 (1993).

${ }^{48}$ S. A. Stepanov, U. Pietsch, and G. T. Baumbach, Z. Phys. B 96, 341 (1995).

${ }^{49}$ G. T. Baumbach, S. Tixier, U. Pietsch, and V. Holý, Phys. Rev. B 51, 16848 (1995).

${ }^{50}$ D. Bahr, W. Press, R. Jebasinski, and S. Mantl, Phys. Rev. B 51, 12223 (1995).

${ }^{51}$ S. A. Stepanov et al., Phys. Rev. B 54, 8150 (1996).

${ }^{52}$ A. M. Afanas'ev et al., Phys. Status Solidi A 86, K1 (1984); 90, 419 (1985).

${ }^{53}$ A. M. Afanas'ev et al., Kristallografiya 31, 1066 (1986) [Sov. Phys. Crystallogr. 31, 630 (1986)].

${ }^{54}$ G. D. Yao, J. Wu, T. Fanning, and M. Dudley, Adv. X-Ray Anal. 35, 247 (1992).

${ }^{55}$ O. G. Melikyan and A. M. Meretukov, Kristallografiya 38, 42 (1993) [Crystallogr. Rep. 38, 311 (1993)].

${ }^{56}$ M. A. Andreeva, K. Rocete, and Y. P. Khapachev, Phys. Status Solidi A 88, 455 (1985).
${ }^{57}$ D. W. Berreman and A. T. Macrander, Phys. Rev. B 37, 6030 (1988).

${ }^{58}$ S. A. Stepanov, Kristallografiya 39, 221 (1994) [Crystallogr. Rep. 39, 182 (1994)].

${ }^{59}$ S. A. Stepanov and R. Köhler, J. Phys. D 27, 1923 (1994).

${ }^{60}$ S. Takagi, Acta Crystallogr. 15, 1311 (1962); J. Phys. Soc. Jpn. 26, 1239 (1969).

${ }^{61}$ D. Taupin, Bull. Soc. Fr. Mineral. Cristallogr. 87, 469 (1964).

${ }^{62}$ F. Abeles. Ann. Phys. (Paris) 3, 504 (1948); 5, 596 (1950).

${ }^{63}$ L. G. Parratt, Phys. Rev. 95, 359 (1954).

${ }^{64}$ W. J. Bartels, J. Hornstra, and D. J. W. Lobeek, Acta Crystallogr., Sect. A: Found. Crystallogr. 42, 539 (1986).

${ }^{65}$ S. M. Durbin and G. C. Follis, Phys. Rev. B 51, 10127 (1995).

${ }^{66}$ T. Takahashi and S. Nakatani, Surf. Sci. 326, 347 (1995).

${ }^{67}$ Y. P. Khapachev and F. N. Chukhovskii, Kristallografiya 34, 776 (1989) [Sov. Phys. Crystallogr. 34, 465 (1989)].

${ }^{68}$ M. A. Andreeva and S. F. Borisova, Kristallografiya 30, 849 (1985) [Sov. Phys. Crystallogr. 30, 493 (1985)].

${ }^{69}$ R. W. James, Solid State Phys. 15, 53 (1963).

${ }^{70}$ B. W. Batterman and H. Cole, Rev. Mod. Phys. 36, 681 (1964).

${ }^{71}$ Z. G. Pinsker, Dynamical Scattering of X-Rays in Crystals (Springer-Verlag, Berlin, 1981).

${ }^{72}$ The sign of $\gamma_{\mathrm{hz}}$ in our definition is inverted with respect to that in usual definitions.

${ }^{73}$ In the case of grazing incidence/exit $u_{n}$ can be treated as the angles between the $\mathrm{x}$-ray waves inside layers and the surface.

${ }^{74}$ A. M. Afanas'ev et al., Acta Crystallogr., Sect. A: Found. Crystallogr. 42, 116 (1986).

${ }^{75}$ I. K. Robinson, Phys. Rev. B 33, 3830 (1986).

${ }^{76}$ A. Caticha, Phys. Rev. B 47, 76 (1993).

${ }^{77}$ R. Colella, Phys. Rev. B 43, 13827 (1991).

${ }^{78}$ P. A. Aleksandrov, A. M. Afanas'ev, and S. A. Stepanov, Poverkhn., Fiz. Khim. Mekh. 8, 9 (1984) [Phys. Chem. Mech. Surf. 3, 2222 (1985)].

${ }^{79}$ V. G. Kohn, J. Mosc. Phys. Soc. 1, 425 (1991).

${ }^{80}$ The amplitudes in a thick substrate can be normalized arbitrarily, since they enter only one equation.

${ }^{81}$ M. Schuster et al., J. Phys. D 28, A206 (1995).

${ }^{82}$ C. Bocchi et al., J. Cryst. Growth 132, 427 (1993).

${ }^{83}$ S. A. Stepanov and R. Köhler, J. Appl. Phys. 76, 7809 (1994).

${ }^{84}$ A. Authier, J. Gronkowski, and C. Malgrange, Acta Crystallogr., Sect. A: Found. Crystallogr. 45, 432 (1989).

${ }^{85}$ A. P. Ulyanenkov, S. A. Stepanov, U. Pietsch, and R. Köhler, J. Phys. D 28, 2522 (1995).

${ }^{86}$ V. M. Kaganer et al., Phys. Rev. B 55, 1793 (1997). 